UNIVERSIDADE DE SÃO PAULO

FACULDADE DE MEDICINA DE RIBEIRÃO PRETO

CLARISSA VILELA RODRIGUES VIEIRA DE CARVALHO DIAS

Ultrassom para monitorização da estimulação ovariana controlada: revisão sistematizada e metanálise de estudos randomizados controlados 


\section{Ultrassom para monitorização da estimulação ovariana controlada: revisão sistematizada e metanálise de estudos randomizados controlados}

Dissertação apresentada à Faculdade de Medicina de Ribeirão Preto da Universidade de São Paulo para obtenção do Título de Mestre em Ciências Médicas.

Área de Concentração: Ginecologia e Obstetrícia.

Orientador: Prof. Dr. Wellington de Paula Martins 
AUTORIZO A REPRODUÇÃO E DIVULGAÇÃO TOTAL OU PARCIAL DESTE TRABALHO, POR QUALQUER MEIO CONVENCIONAL OU ELETRÔNICO, PARA FINS DE ESTUDO E PESQUISA, DESDE QUE CITADA A FONTE.

\section{FICHA CATALOGRÁFICA}

Dias, Clarissa Vilela Rodrigues Vieira de Carvalho

Ultrassom para monitorização da estimulação ovariana controlada: revisão sistematizada e metanálise de estudos randomizados controlados. I Clarissa Vilela Rodrigues Vieira de Carvalho Dias / Orientador: Wellington de Paula Martins. Ribeirão Preto, 2015.

63p.: 9il.; $30 \mathrm{~cm}$

Dissertação de Mestrado apresentada à Faculdade de Medicina de Ribeirão Preto/USP. Área de Concentração: Ginecologia e Obstetrícia.

1. Ultrassonografia; 2. Técnicas de reprodução assistida; 3. Indução de ovulação; 4. Revisão sistematizada; 5. Metanálise. 
Aluna: Clarissa Vilela Rodrigues Vieira de Carvalho Dias

Título: Ultrassom para monitorização da estimulação ovariana controlada: revisão sistematizada e metanálise de estudos randomizados controlados.

Dissertação apresentada à Faculdade de Medicina de Ribeirão Preto da Universidade de São Paulo, para a obtenção do título de Mestre em Ciências Médicas.

Área de Concentração: Ginecologia e Obstetrícia.

Aprovada em:

\author{
Banca Examinadora
}

Prof. Dr.

Instituição:

Assinatura:

Prof. Dr.

Instituição:

Assinatura:

Prof. Dr.

Instituição:

Assinatura: 


\section{Dedicatória}

Dedico este trabalho aos meus amores: minha família!

À minha avó Nena, exemplo de trabalho, inteligência, retidão e força, sem deixar de lado a ternura. Meu eixo quando precisei, sempre vibrando pelas minhas conquistas, mesmo quando tão pequenas. Me ensinou o valor do estudo logo cedo, sempre tinha um livro para me presentear ou um recorte de jornal com um artigo interessante, sua forma de compartilhar informações. Pena ser mortal, me deixou aos 92 anos, como sinto sua falta. Mas este trabalho também é fruto do seu, na minha educação. Por isso te dedico vovó Nena, minha Dissertação de Mestrado. Obrigada!

Ao meu filho Bento, o maior amor de todos, a forma mais pura e única. Você me faz acreditar em milagres todos os dias. Adoro participar de suas descobertas, me deu a melhor função de todas, a de MÃE.

Ao meu companheiro Gabriel. Do latin "cum panis"; aquele com quem dividimos o pão. Aquele que confiamos o suficiente para sentar em nossa mesa e dividir nossas ideias, vitórias, derrotas ou um simples pedaço de pão. É aquele que nos diverte, conforta, acompanha, protege, ouve, fala, aconselha, carrega, alimenta, nas horas alegres ou tristes, durante e depois de nossa etérea vida. Acho que é isso, meu amor.

Aos meus pais, que sempre me apoiaram, comemoraram minhas vitórias e estiveram fortes e presentes quando parecia que tudo não iria dar certo. Toda minha gratidão!

A Mel e ao Costela, meus queridos cachorros, que fizeram companhia ao Gabriel quando não pude estar presente.

À minha sogra querida Zizi, com muito carinho.

À minha irmã Heloisa, que me lembra que a vida é para ser leve e divertida.

Ao meu querido tio Omar. 


\section{Agradecimentos}

Minha gratidão,

Ao meu orientador, Wellington de Paula Martins. Um amigo que conheci nessa jornada e que mostrou a importância da formação acadêmica. Um professor no sentido mais amplo. Agradeço a confiança e ajuda. Agradeço também as críticas, sempre me impulsionando a melhorar. Obrigada por me fazer compreender a importância de nossa profissão e dar importância às coisas que realmente fazem diferença.

Agradeço a amiga Carol Nastri, pela participação essencial neste trabalho.

À minha colega e amiga Danielle M. Teixeira, que me inspira a ser melhor profissional e aluna mais comprometida. Me encanto com sua disciplina e inteligência. Que saudades e que falta você me faz.

À Marisa, Lilian, Sônia, Didi, Devanir, obrigada pela ajuda.

Aos amigos que fiz na residência, obrigada pela companhia, pela alegria, pelas pizzas, trocas de plantões e substituições.

À Equipe do Setor de Reprodução Humana do HC, pelos ensinamentos, não só profissionais, mas de amizade e de vida.

À Equipe do Departamento de Ginecologia e Obstetricia da Faculdade de Medicina de Ribeirão Preto da Universidade de São Paulo, obrigada pela importante participação na minha formação. 
Apoio Financeiro

Este estudo recebeu o apoio financeiro da Fundação de Amparo à Pesquisa do Estado de São Paulo - FAPESP, por meio de concessão de bolsa de Mestrado às alunas Clarissa V. R. V. de Carvalho Dias e Danielle Medeiros Teixeira. 
DIAS, C.V.R.V.C. Ultrassom para monitorização da estimulação ovariana controlada: revisão sistematizada e metanálise de estudos randomizados controlados. 2015. 63f. Dissertação (Mestrado) da Faculdade de Medicina de Ribeirão Preto da Universidade de São Paulo. Ribeirão Preto-SP. 2015.

Justificativa: As técnicas de reprodução assistida (TRA), usadas para o tratamento de infertilidade/subfertilidade, incluem manipulação in vitro de oócitos e esperma, ou embriões, com o objetivo de alcançar gravidez e nascimentos vivos. O recrutamento de múltiplos folículos é fundamental para o aumento das taxas de gravidez, e isso é alcançado por meio do estímulo ovariano controlado (EOC). A monitorização do EOC é realizada por contagem de folículos ovarianos e medidas ultrassonográficas, associadas ou não à dosagem hormonal. Justificase monitorar a fase folicular para decisões a respeito da dose de gonadotrofinas administradas, detecção do risco de ocorrência da síndrome de hiperestímulo ovariano $(\mathrm{SHO})$ e do planejamento do triggering da maturação final; porém a necessidade da monitorização intensiva da EOC é controversa, pois a combinação dos métodos consome mais tempo, recursos e está associada com maior desconforto para a paciente. Objetivo: Avaliar a eficácia e segurança da monitorização da EOC em ciclos de reprodução assistida, usando somente ultrassonografia (US). Métodos de busca: As buscas por estudos randomizados foram realizadas nos principais bancos de dados eletrônicos. Além disso, foram examinadas, manualmente, as listas de referências dos estudos incluídos em revisões semelhantes. A última busca eletrônica foi realizada em 12 de março de 2015. Critérios de Seleção: Apenas estudos verdadeiramente randomizados, que comparassem a monitorização do EOC por US associado à dosagem hormonal e US isoladamente, monitorização do EOC por US2D e US3D, bem como US2D e telemonitorização endovaginal operada pela própria paciente (SOET), foram considerados elegíveis. Os estudos que permitiam a inclusão de uma mesma paciente duas vezes foram incluídos apenas se os dados do primeiro ciclo estivessem disponíveis. Coleta e Análise de Dados: Dois revisores avaliaram, independentemente, a elegibilidade, extração de dados e os riscos de viéses dos estudos incluídos. Quaisquer discordâncias foram resolvidas em consulta com um terceiro revisor. Quando necessário, os autores dos estudos incluídos foram contatados para maiores informações. Resultados: Foram selecionados 1717 registros, 10 dos quais eram elegíveis. Nenhum estudo relatou nascidos vivos. Seis estudos compararam a monitorização do EOC por US isolada com US associada à dosagem hormonal. Os intervalos de confiança (IC) foram extensos e não permitiram concluir a existência de benefício nem prejuízo associado ao uso de US isolada, em relação aos desfechos SHO (odds ratio $\mathrm{OR}=1.03, \mathrm{IC} 95 \% 0.48$ a $2.18, \mathrm{p}=0.95$ ) e abortamento (risco relativo - $\mathrm{R} R=0.37$, IC95\% 0.07 a 1.79, $p=0.21$ ). Para gravidez clínica, o IC foi compatível com pequeno benefício a pequeno prejuízo ( $R R=0.96, I C 95 \% 0.80$ a $1.16, p=0.70)$. Para número de oócitos captados, o IC foi compatível com apreciável benefício a não efeito (Diferença média $\mathrm{MD}=0.92$ oócitos captados, $\mathrm{Cl} 95 \%-0.19$ a 2.04 , $p=0.70$ ). Dois estudos compararam US3D e US2D e os IC foram extensos e não permitiram concluir pela existência de benefício nem prejuízo associado à monitorização por US3D para os desfechos: gravidez clínica $(R R=1.00, I C 95 \%$ 0.58 a 1.73) e número de oócitos captados (MD= -0.37 oócitos, IC95\% -3.63 a 
2.89). Apenas um estudo comparou monitorização por US2D convencional com SOET, e o IC observado foi amplo e não permitiu concluir pela existência de benefício nem prejuízo associado à SOET, considerando se gravidez clínica ( RR $=0.95$, IC $95 \% 0.52$ a 1.75) e número de oócitos captados (MD=0.50, Cl 95\% 2.13 a 3.13). Conclusão: No que concerne à eficácia, as evidências atuais sugerem que monitorizar $\mathrm{O}$ EOC apenas com US não deva alterar, substancialmente, as chances de se alcançar gravidez clínica. O número de oócitos captados é similar ao se comparar com a monitorização por US associada à dosagem hormonal. Quanto à segurança, também não houve aumento no risco de desenvolvimento de SHO. Contudo, a interpretação dos resultados deve ser realizada com cautela, já que para todos os desfechos e todas as comparações, os dados disponíveis são inconclusivos, pois a qualidade de evidência foi comprometida por imprecisão e falha dos estudos em relatar a metodologia aplicada. Por isso acredita-se que serão necessários mais estudos avaliando o procedimento ideal para monitorização da EOC

Palavras-chave: Ultrassonografia, Técnicas de reprodução assistida, Indução da ovulação, Revisão sistematizada, Metanálise. 
DIAS, C.V.R.V.C. Ultrasound for monitoring controlled ovarian stimulation: a systematic review and meta-analysis of randomized controlled trials. 2015. 63f. Dissertation (Master) Ribeirão Preto Medical School, University of São Paulo. Ribeirão Preto-SP. 2015.

Background: The assisted reproductive techniques (ART) for the treatment of infertility/subfertility, include in vitro handling of both human oocytes and sperm or of embryos with the objective of achieving pregnancy and live birth. The recruitment of multiple follicles is often necessary for better results in pregnancy rates and it's achieved by performing controlled ovarian stimulation (COS). COS monitoring is performed by ovarian follicle counting and ultrasonography measurements and / or hormones dosage. It is appropriate to monitor the follicular phase for decisions regarding administered of gonadotropin dose, to assess the risk of ovarian hyperstimulation syndrome (OHSS), to determine the best time to trigger final follicular maturation. However, the need for intensive COS monitoring is controversial: the combination of the methods adds costs and discomfort for the woman who is undergoing ART and requires additional time. Objectives: To evaluate the efficacy and safety of monitoring controlled ovarian stimulation by ultrasound in assisted reproduced tecniques. Search Methods: The searches for randomized controlled trials (RCT) were performed in the main electronic databases; in addition, we hand searched the reference lists of included studies and similar reviews. We performed the last electronic search on March 29, 2015. Selection Criteria: Only truly randomized controlled trials comparing COS monitoring by ultrasonography and/or hormonal assessment, as studies comparing COS monitoring by 2DUS and 3DUS were considered eligible. We included studies that permitted the inclusion of the same participant more than once (cross-over or 'per cycle' trials) only if data regarding the first treatment of each participant were available. Data Collection and Analysis: Two reviewers independently performed study eligibility, data extraction, and assessment of the risk of bias and we solved disagreements by consulting a third reviewer. We corresponded with study investigators in order to resolve any queries, as required. Results: The search retrieved 1717 records; ten studies were eligible. No study reported live birth. Six studies compared US only vs. US + Hormones. The confidence intervals $(\mathrm{Cl})$ were large and did not allow us conclude benefit or harm associated with the US Only for both OHSS (Odds ratio - OR=1.03, 95\% $\mathrm{Cl} 0.48$ to 2.18, $\mathrm{P}=0.95$ ), and miscarriage (relative risk - $\mathrm{RR}=0.37,95 \% \mathrm{Cl} 0.07$ to 1.79 , $\mathrm{P}=0.21$ ). For clinical pregnancy, the $\mathrm{Cl}$ was compatible with small benefit to small harm $(R R=0.96,95 \% \mathrm{Cl} 0.80$ to $1.16, P=0.70)$. For the number of oocytes retrieved, the $\mathrm{Cl}$ was compatible with appreciable benefit to no effect (Mean difference - $\mathrm{MD}=0.92$ oocytes, $95 \% \mathrm{Cl}-0.19$ to $2.04, \mathrm{P}=0.70)$. Two studies compared 3DUS vs. 2DUS: the confidence intervals $(\mathrm{Cl})$ were large and did not allow us conclude benefit or harm associated with 3DUS regarding clinical pregnancy $(R R=1.00, C 195 \% 0.58$ to 1.73$)$ and number of oocytes retrieved (MD= -0.37 oocytes, $95 \% \mathrm{Cl}-3.63$ to 2.89 ). One study compared 2DUS vs. SOET, the $\mathrm{Cl}$ was large and did not allow us conclude benefit or harm associated with SOET regarding clinical pregnancy $(\mathrm{RR}=0.95,95 \% \mathrm{Cl} 0.52$ a 1.75$)$ and number of oocytes retrieved (MD=0.50, $95 \% \mathrm{Cl}-2.13$ a 3.13). Authors' Conclusions: Regarding effectiveness, current evidence suggests that monitoring COS only by 
US only should not change substantially the chances of achieving clinical pregnancy. The number of retrieved oocytes is similar to compare with the monitoring by US associated with hormonal assessment. security also seems not to increase the risk of developing OHSS. However the interpretation of results should be performed with caution, since for all outcomes and comparisons, the available data are inconclusive because the quality of evidence was compromised by inaccuracy and poor reporting of study methodology. So we believe that further studies evaluating the ideal procedure for monitoring the COS are needed.

Keywords: Ultrasonography; Assisted reproduction techniques; Ovulation induction; Systematic review; Metanalysis. 
LISTA DE FIGURAS 
Figura 1: Fluxograma da seleção dos estudos 40

Figura 2: Síndrome de hiperestímulo ovariano (SHO)- Forest Plot: Comparação US isolada versus US + dosagem Hormonal.

Figura 3: Gravidez clínica - Forest Plot: comparação US Isolada versus US + dosagem Hormonal.

Figura 4: Abortamento por gravidez clínica - Forest Plot: comparação US Isolada versus US + dosagem Hormonal. .

Figura 5: Número de oócitos captados- Forest Plot: comparação US Isolada versus US + dosagem Hormonal

Figura 6: Gravidez clínica - Forest Plot: comparação US3D versus US2D.

Figura 7: Número total de oócitos captados - Forest Plot: comparação US3D versus US2D.

Figura 8: Gravidez clínica - Forest Plot: comparação SOET versus US2D 49

Figura 9: Número total de oócitos captados - Forest Plot: comparação SOET versus US2D 
LISTA DE TABELAS 
Tabela 1: Característica dos estudos excluídos .................................... 43

Tabela 2: Detalhes dos estudos incluídos na comparação US + E2 vs. US ..... 51

Tabela 3: Detalhes dos estudos incluídos na comparação US2D vs. US3D .... 52

Tabela 4: Detalhes do estudo incluído na comparação US2D vs. SOE ............ 53 
CENTRAL: Cochrane Central Register of Controlled Trials

CFA: $\quad$ Contagem de folículos antrais

CINAHL: $\quad$ Cumulative Index to Nursing and Allied Health Literature

CON: $\quad$ Refere-se à coautora - Carolina Oliveira Nastri

CVRV: $\quad$ Refere-se à autora - Clarissa V. R. Vieira

DARE: Cochrane Library for Database of Abstracts of Reviews of Effects

DMT: $\quad$ Refere-se à coautora - Danielle Medeiros Teixeira

E2: $\quad$ Estradiol

ECR: $\quad$ Ensaio clínico randomizado

EOC: Estímulo ovariano controlado (do inglês controlled ovarian stimulation)

FIV: $\quad$ Fertilização in vitro (do inglês in vitro fertilization)

FSH: hormônio folículo-estimulante (do inglês follicle-stimulating hormone)

GIFT: $\quad$ Trasnferência tubária de oocitos e espermatozoides

GnRH: hormônio liberador de gonadotrofina (do inglês gonadotropinreleasing hormone

GRADE: Grading of Recommendations Assessment, Development and Evaluation

hCG: gonadotrofina coriônica humana (do inglês human chorionic gonadotropin)

IC: Intervalo de confiança

ICSI: Injeção intracitoplasmática de espermatozoide

IMC: $\quad$ Índice de massa corpórea (do inglês body mass índex)

ITT: Intention to treat

IUI: Inseminação intrauterina

LAD: $\quad$ Refere-se à autora - Lilian A. D'assunção 


\section{LH: Hormônio luteinizante}

LILACS: Literatura Latino-Americana e do Caribe em Ciências da Saúde

MAPB: $\quad$ Refere-se à coautora - Mariana Alves Parente Barbosa

MD: $\quad$ Diferença média (do inglês mean difference)

MDSG: $\quad$ Cochrane Menstrual Disorders and Subfertility Group

MEDLINE: Medical Literature Analysis and Retrieval System Online

NNH: $\quad$ Number needed to harm

NNT: $\quad$ Number needed to treat

OMS: $\quad$ Organização Mundial da Saúde

OR: $\quad$ Odds ratio

RCT: $\quad$ Randomized controlled trials

r-FSH: hormônio folículo-estimulante recombinante (do inglês recombinant follicle-stimulating hormone)

r-hCG: Recombinant human chorionic gonadotropin

RR: $\quad$ Risco relativo

SHO: Síndrome de hiperestímulo ovariano (do inglês ovarian hyperstimulation syndrome)

SIGN: $\quad$ Scottish Intercollegiate Guidelines Network

SOET- Telemonitorização endovaginal operada pela própria paciente

SonoAVC: Sono Automated Volume calculation (cálculo automatizado de volume por ultrassonografia)

TE: $\quad$ Trasnferência de embriões

TRA: $\quad$ Técnicas de reprodução assistida

US: $\quad$ Ultrassonografia

US2D: $\quad$ Ultrassonografia em duas dimensões

US3D: $\quad$ Ultrassonografia em três dimensões 
WPM: $\quad$ Refere-se ao coautor e orientador - Wellington de Paula Martins

ZIFT: $\quad$ Trasnferência tubária de oocitos e zigotos

Observação: Pela similaridade de algumas abreviaturas em português e pelo fato de muitas das abreviaturas em inglês serem amplamente utilizadas na prática clínica, optou-se por se manterem aquelas utilizadas na literatura internacional, para facilitar o entendimento do leitor. 


\section{SUMÁRIO}

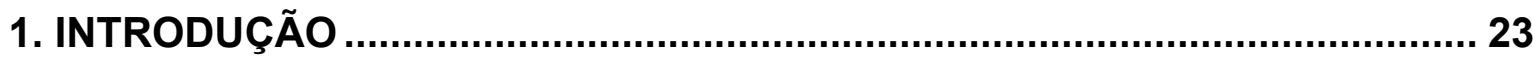

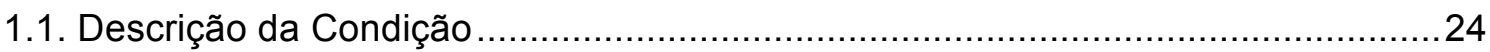

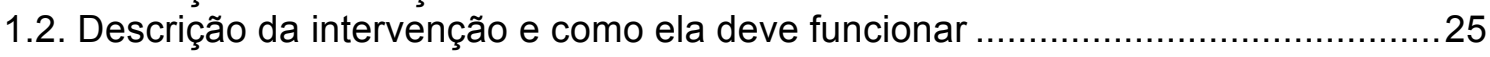

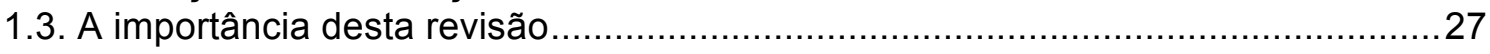

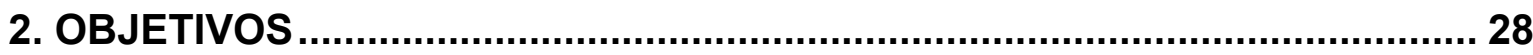

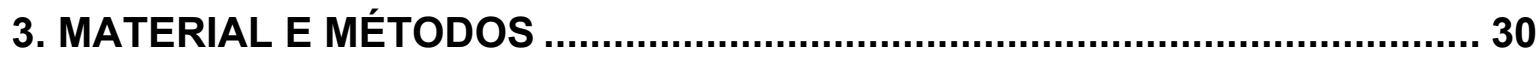

3.1. Protocolo e registro ...................................................................................

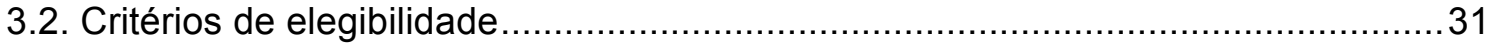

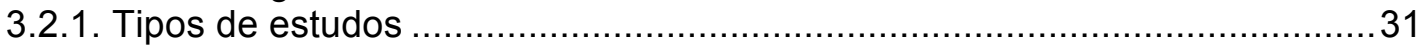

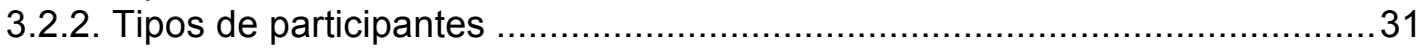

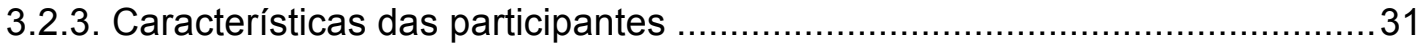

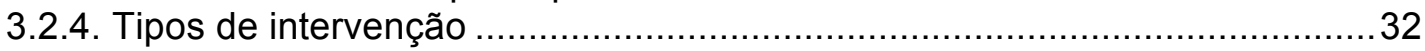

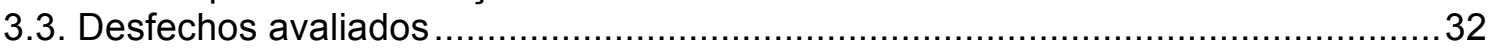

3.4. Métodos de busca para identificação dos estudos .............................................32

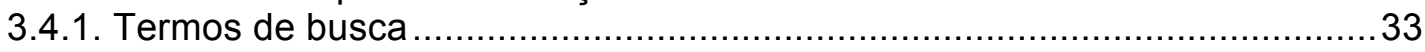

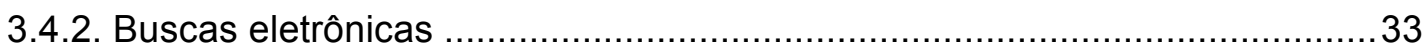

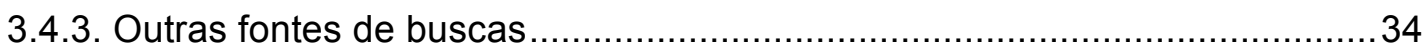

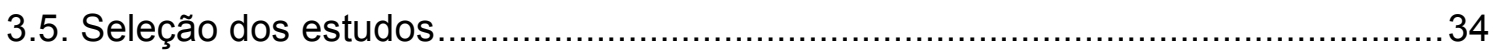

3.5.1. Processo de coleta de dados ...............................................................34

3.6. Análise do risco de viés dos estudos incluídos.....................................................35

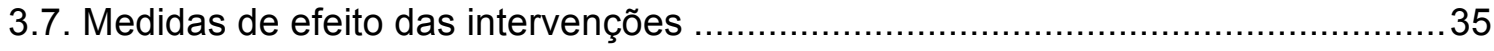

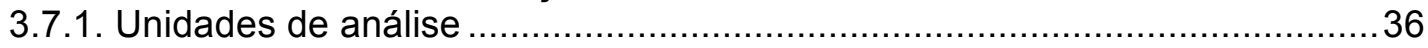

3.7.2. Avaliação da heterogeneidade e avaliação da sensibilidade ..........................36

3.7.3. Risco de viés entre os estudos.............................................................37

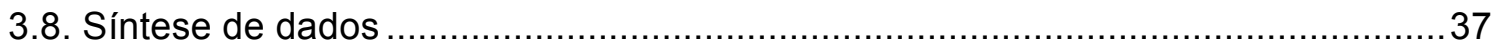

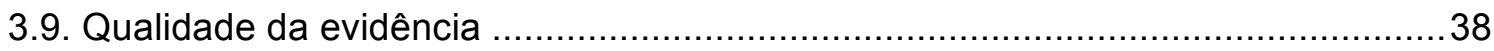

4. RESULTADOS

4.1. Resultados da seleção de estudos...............................................................40

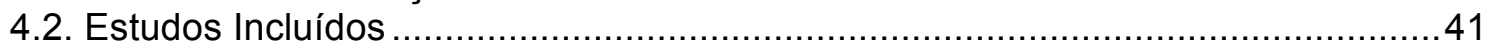

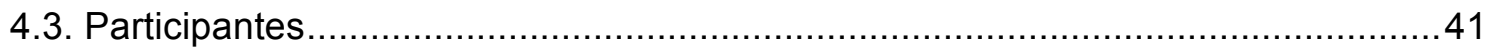

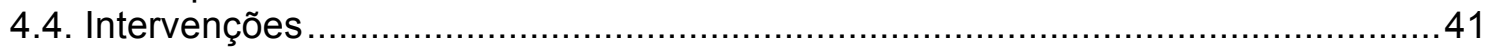

4.5. Resultados das análises dos estudos incluídos ............................................42

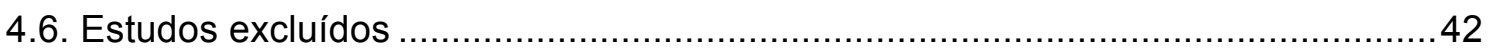

4.7. Estudos aguardando classificação ..................................................................4

4.8. Risco de viés nos estudos inclú́dos ............................................................. 43

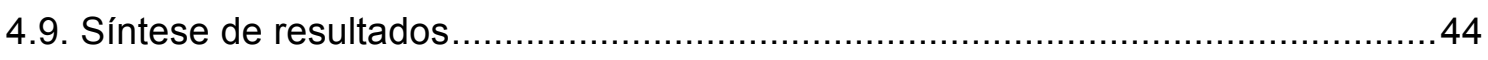

4.9.1. Comparação US isolado vs. US + dosagem hormonal ...............................44

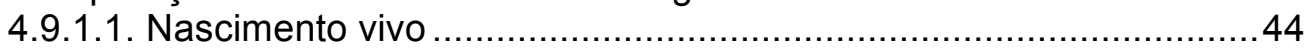

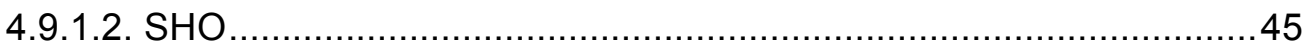

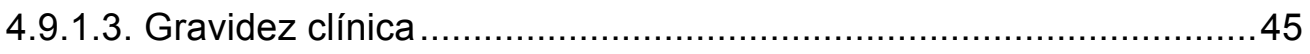

4.9.1.4. Abortamento por gravidez clínica.................................................46

4.9.1.5. Número total de oócitos captados ................................................46

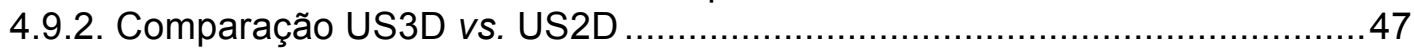

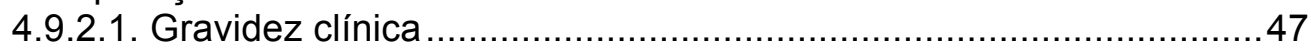


4.9.2.2. Número total de oócitos captados ................................................48

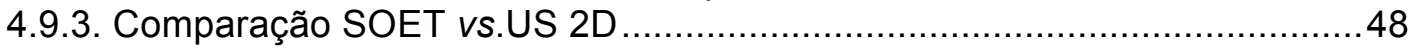

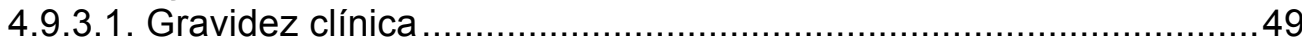

4.9.3.2. Número total de oócitos captados ............................................49

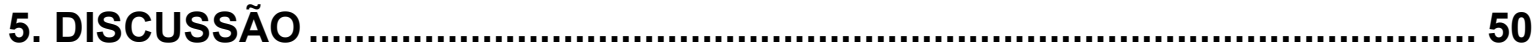

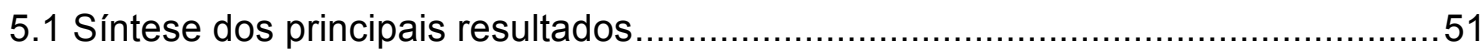

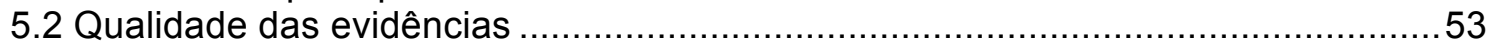

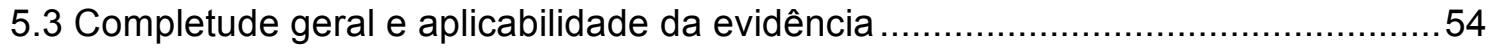

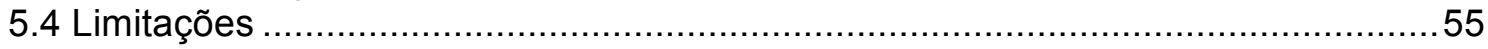

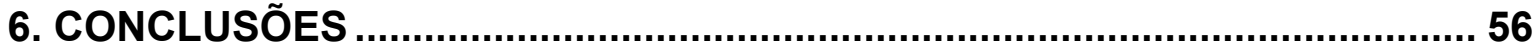

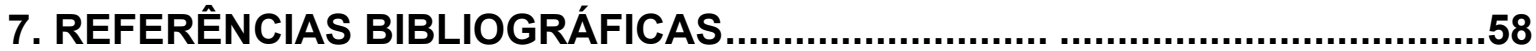

ANEXO DE PUBLICAÇÃO 
1- INTRODUÇÃO 


\subsection{Descrição da condição}

As técnicas de reprodução assistida (TRA) são amplamente utilizadas para o tratamento de infertilidade/subfertilidade (Gnoth et al., 2005). Subfertilidade descreve uma forma de redução da fertilidade com tempo prolongado de não concepção indesejada. Infertilidade poderia ser usada como sinônimo de esterilidade em que pode ocorrer gestação espontânea apenas esporadicamente. Clinicamente, subfertilidade é a disfunção do sistema reprodutivo em que não ocorre gestação após 12 meses de relações sexuais regulares desprotegidas no período fértil dos ciclos menstruais. Estima-se que essas condições afetem entre $10 \%$ e $15 \%$ dos casais em idade reprodutiva (Evers, 2002).

As TRA incluem todos os tratamentos e procedimentos que requerem a manipulação in vitro de oócitos e de esperma, ou de embriões, com o objetivo de alcançar gravidez e nascimentos vivos. A fertilização in vitro (FIV) é a primeira e a mais comum delas. Contudo, também incluem fertilização assistida, como a injeção intracitoplasmática de espermatozoides (ICSI), indicada para casais com fator masculino de infertilidade, transferência tubária de oócitos e espermatozoides (GIFT), ou de zigotos (ZIFT), ou embriões (TE), criopreservação de gametas e embriões, doação de oócitos e embriões e gestação compartilhada. Não compreende a inseminação intrauterina (IUI) (Zegers-Hochschild et al., 2009).

Nas TRA é desejável o recrutamento de múltiplos folículos por meio do estímulo ovariano controlado (EOC), com o objetivo de aumentar o número de oócitos captados, número de embriões resultantes e, consequentemente, as chances de gravidez (Macklon et al., 2006; Wiser et al., 2012). Esta é uma etapa importante, considerando-se que a taxa estimada de gestação por ciclo com o estímulo ovariano mínimo é de cerca de 10\% (Pelinck et al., 2006) e sobe para $33 \%$ quando é realizado o EOC padrão (Gnoth et al., 2011). Na maioria dos protocolos é administrado agonista ou antagonista de GnRH (hormônio liberador de gonadotrofina - do inglês gonadotropin-releasing hormone) para a supressão da secreção endógena de hormônio folículo-estimulante (FSH) e hormônio luteinizante (LH), e em seguida, se administra $\mathrm{FSH}$, visando o desenvolvimento multifolicular. Confirmado o crescimento de vários folículos por ultrassonografia 
(US) e/ou por dosagem hormonal (estradiol - E2) é, então, indicada uma única dose de hCG (gonadotrofina coriônica humana - do inglês human chorionic gonadotropin) para substituir o pico de LH na maturação oocitária final (Macklon; Fauser, 2003). Identificar o desenvolvimento adequado e avaliar o melhor momento para a administração do hCG não é uma tarefa simples.

Ao estimular o crescimento de mais folículos para melhores resultados das TRA, o EOC aumenta o risco de uma complicação iatrogênica grave e de ameaça potencial à vida: a síndrome de hiperestímulo ovariano (SHO). A SHO consiste em uma resposta sistêmica exagerada ao estímulo ovariano, caracterizada por amplo espectro de manifestações clínicas e laboratoriais. Ocorre o aumento da permeabilidade capilar e do volume dos ovários. Há perda de fluídos dos vasos para o terceiro espaço, resultando em hipovolemia. Pode ser classificada em leve, moderada ou severa, de acordo com o grau de distensão abdominal, aumento de volume ovariano, complicações respiratórias (derrame pleural), hemodinâmicas (oligúria, ascite, hemoconcentração, fenômenos tromboembólicos) e metabólicas. A SHO severa requer internação hospitalar e pode ocorrer em até $3 \%$ dos ciclos. Entre as pacientes de alto risco (jovens, IMC baixo, aquelas que receberam altas doses de gonadotrofinas, as que apresentam aumento dos níveis de estradiol sérico e que tiveram episódio prévio de SHO) pode chegar a 20\% (Nastri et al., 2010; Cantwell et al., 2011; Nastri et al., 2015).

\subsection{Descrição da intervenção e como ela deve funcionar}

A contagem e a medida dos folículos ovarianos por US, bem como a dosagem dos níveis hormonais, particularmente a concentração de E2 sérico, são ferramentas tradicionalmente utilizadas para monitorização da indução da ovulação com gonadotrofinas. Por meio da US é possível observar o desenvolvimento dos folículos, determinando-se o melhor momento para a administração da hCG para a indução da maturação final. O seguimento ultrassonográfico dos folículos combinado com dosagens séricas de E2 pode ser útil na decisão de postergar a administração do hCG, garantindo a segurança do tratamento, a fim de minimizar a ocorrência e a severidade da SHO (Golan et al., 
1994). Ao monitorar a EOC, o clínico é capaz de decidir por: 1) cancelamento precoce dos ciclos sem resposta ovariana adequada, poupando gastos desnecessários de recursos; 2) o momento mais apropriado para maturação folicular final (trigger), para maximizar o número de oócitos maduros captados; 3) avaliação do risco de SHO, importante quando são consideradas estratégias recentes úteis na prevenção dessa indesejada complicação, como por exemplo, o uso da cabergolina (Tang et al., 2012), ou a substituição da hCG por agonista de GnRH no trigger da maturação folicular final (Humaidan et al., 2012). Acredita-se que a combinação de US e dosagem hormonal possa fornecer informações adicionais ao médico; evitando-se, assim, tomadas de decisões equivocadas a partir do erro de um dos métodos. Por outro lado, a necessidade de monitorização intensiva do EOC é controversa, pois apesar de ser um marcador funcional da foliculogênese as concentrações de E2, não estão sempre correlacionadas com o crescimento folicular, especialmente no caso de indução da ovulação. Alguns autores alegam que a combinação dos métodos consome mais tempo e recursos e está associada a maior desconforto para a paciente e sugerem monitoração simplificada dos ciclos de FIV, sem afetar os resultados e nem aumentar a incidência de SHO (Abdalla et al., 1989; Roest et al., 1995). Outros propõem a monitorização do EOC apenas por US, demonstrando em seus estudos eficácia e segurança similares àquelas observadas com a monitoração combinada (Lass, 2003).

Publicações atuais propõem a substituição da monitorização tradicional realizada por US2D pela US tridimensional (US3D). A avaliação das dimensões foliculares foi descrita em meados dos anos 90 (Kyei-Mensah et al., 1996). Recentemente, têm sido estudadas medidas semiautomáticas de volume ou diâmetro folicular por US3D, por meio da medida de volume baseada em US usando sonoAVC (sono automated volume calculation). O SonoAVC (GE Medical Systems, Zipf, Áustria) é um software desenvolvido para cálculos automáticos de volume de áreas preenchidas por fluído. Esse método demonstrou boa concordância com a US convencional e requer menos tempo, sendo particularmente vantajoso na presença de multiplos folículos. Outras vantagens potenciais são: o uso do diâmetro baseado no volume, ao invés da média entre 
diferentes diâmetros, reduz a variabilidade de medidas em folículos não esféricos; em TRA, o volume folicular é um indicador importante de maturidade oocitária, melhores resultados correlacionam com oócitos obtidos a partir de folículos medindo entre 12 e 24 mm considerando-se que o folículo é esférico, porém a maioria tem forma elíptica. Sendo assim, o US3D parece ter maior acurácia; diminui o risco de perda ou de dupla contagem de folículos (Deutch et al., 2009; Vandekerckhove et al., 2013).

Pensando na simplificação da monitoração do EOC e em tornar as TRA menos desgastantes para as pacientes, foi proposta a telemonitorização endovaginal operada pela própria paciente (SOET) (Gerris; De Sutter, 2010), que consiste na possibilidade da paciente ou seu parceiro realizarem suas próprias US de monitoração do EOC em casa. Para isso foi desenvolvida uma sonda vaginal portátil, domiciliar, fácil de usar, que grava as imagens, bem como um software para transferência das imagens, via internet, ao centro de reprodução para serem analisadas pelo médico. Esse método, porém, até o momento, só está disponível para pesquisa.

\subsection{A importância desta revisão}

Considerando que os benefícios de monitorar a EOC já estão bem estabelecidos na literatura e a monitorização contínua é praticada em todo o mundo, o método mais apropriado deve ser definido. As recomendações existentes, até o momento, para essa metanálise são ambíguas. $\mathrm{Na}$ última revisão sistematizada e metanálise publicada e atualizada (Kwan et al., 2008, 2014), os autores não encontraram evidências de que a monitorização da EOC combinada é superior àquela realizada por US isolada em relação aos nascimentos vivos e gravidez clínica; contudo sugerem que o uso dos métodos combinados para monitorizar o EOC, seja uma boa prática, já que as evidências de prevenção de SHO não foram avaliadas adequadamente pelos estudos randomizados controlados (RCTs) incluídos. A atualização da revisão sistematizada prévia é interessante porque novos estudos sobre o assunto foram publicados, desde então, e a precisão das estimativas pode ter melhorado. 
2. OBJETIVOS 
Os objetivos deste estudo foram avaliar a eficácia e a segurança de se monitorar o EOC por meio de US, com ou sem dosagem hormonal (E2), em mulheres submetidas a TRA, bem como comparar os diferentes métodos ultrassonográficos (US2D, US 3D e SOET), por meio da avaliação dos seguintes desfechos:

- Nascimentos vivos;

- Gravidez clínica;

- Número de oócitos captados;

- SHO. 
3. MATERIAL E MÉTODOS 


\subsection{Protocolo e registro}

O protocolo do presente estudo foi registrado no PROSPERO (http://www.crd.york.ac.uk/prospero/): CRD42012003297.

\subsection{Critérios de elegibilidade}

\subsubsection{Tipos de estudos}

Apenas ensaios clínicos randomizados (ECR), que comparassem o uso de US com US associada à dosagem hormonal ou diferentes técnicas ultrassonográficas para monitorização do EOC, foram considerados elegíveis. Ensaios clínicos "quasi" ou "pseudo" randomizados não foram incluídos (Martins et al., 2011; Nastri et al., 2012). Os estudos que permitiram a inclusão de um mesmo participante mais de uma vez (estudos cross-over ou "por ciclo") foram incluídos apenas se: 1) estivessem disponíveis os dados contendo o primeiro ciclo de cada participante; ou 2) os ciclos consecutivos de cada participante fossem realizados num mesmo grupo: em todos os casos, o denominador foi considerado o número de mulheres alocadas (não o número de ciclos), porém estudos desse tipo não foram identificados.

\subsubsection{Tipos de participantes}

As participantes dos estudos foram mulheres submetidas a TRA.

\subsubsection{Características das participantes}

Foi avaliado o número de mulheres que participaram de cada estudo, a média de idade e as causas de infertilidade. 


\subsubsection{Tipos de intervenção}

Foram incluídos estudos que reportassem mulheres submetidas a diferentes métodos de monitorização do EOC, sendo que a monitorização por US estava presente em pelo menos um dos grupos:

- Monitorização da EOC por US associada à dosagem hormonal (grupo controle), $\mathrm{Ou}$

- Monitorização da EOC somente por US (grupo da intervenção), Ou

- Monitorização da EOC realizada por meio US2D (grupo controle), $\mathrm{Ou}$

- Monitorização da EOC realizada por US3D (grupo da intervenção), $\mathrm{Ou}$

- SOET (grupo da intervenção).

\subsection{Desfechos avaliados}

- Desfecho primário: Nascimentos vivos por mulher alocada; SHO por mulher alocada;

- Desfechos secundários: gravidez clínica por mulher alocada, número de oócitos captados (maduros ou totais).

A taxa de implantação não foi incluída nesta metanálise devido ao denominador para esse desfecho, número embriões transferidos não serem randomizados. No entanto, essas informações, quando presentes, foram inseridas nas características dos estudos incluídos.

\subsection{Métodos de busca para identificação dos estudos}

A estratégia de busca foi desenvolvida em conjunto com a coordenadora de busca de estudos da Cochrane Menstrual Disorders and Subfertility Group (MDSG). 


\subsubsection{Termos de busca}

Foram utilizados termos de busca para US, associados a termos para EOC, ou TRA associados a termos para RCT. As seguintes palavras-chave foram utilizadas: (ultrasonography), ou (ultrasound), ou (ovarian stimulation), ou (ovarian hyperstimulation), ou (ovulation induction), ou (controlled ovarian) ou (IVF), ou (ICSI), ou (in vitro fertilization), ou (intracytoplasmic sperm injection), ou (assisted reproduction), ou (embryo transfer), ou (embryo deposition), ou (embryo replacement) ou (blastocyst). Foram inseridos filtros para estudos randomizados controlados, e ajustados para cada base de dados, quando necessário. Não se restringiram datas de plublicações ou idiomas para a pesquisa.

\subsubsection{Buscas eletrônicas}

As buscas eletrônicas foram realizadas em 15 de abril de 2013 e atualizadas em 12 de março de 2015. Buscaram-se ensaios clínicos randomizados nos seguintes bancos de dados eletrônicos:

- Cochrane Central Register of Controlled Trials (CENTRAL);

- Cumulative Index to Nursing and Allied Health Literature (CINAHL) (www.ebscohost.com/cinahl/);

- Embase. Essa busca foi combinada a filtros para estudos desenvolvidos pelo Scottish Intercollegiate Guidelines Network (SIGN) (http://www.sign.ac.uk/ methodology/filters.html);

- Literatura Latino-Americana e do Caribe em Ciências da Saúde (LILACS) (http://regional.bvsalud.org/php/ index.php?lang=en);

- Medical Literature Analysis and Retrieval System Online (MEDLINE),

- PsycINFO.

Também foram realizadas buscas por protocolos de estudos em andamento nos seguintes bancos de registros de estudos clínicos:

- Current Controlled Trials (www.controlled-trials.com);

- ClinicalTrials.gov (http://clinicaltrials.gov/ct2/search); 
- The World Health Organization International Trials Registry Platform search portal (http://apps.who.int/trialsearch/Default.aspx).

\subsubsection{Outras fontes de buscas}

Os autores realizaram buscas manuais baseadas nas listas de referências dos estudos incluídos e das revisões relacionadas ao tema, procurando por registros adicionais de estudos potencialmente elegíveis.

\subsection{Seleção dos estudos}

A avaliação de elegibilidade foi realizada em duas etapas. Os títulos e resumos dos estudos encontrados foram revisados por dois autores, independentemente, (CVRV e LAD), checados para duplicatas, que usaram os critérios de inclusão pré-estabelecidos. Discordâncias foram resolvidas por consulta a um terceiro revisor e orientador deste estudo (WPM). Esses revisores (CVRV e LAD) recuperaram os textos completos dos estudos considerados potencialmente elegíveis para inclusão. Dois autores (WPM e CON) avaliaram, independentemente, a elegibilidade desses estudos. Discordâncias foram resolvidas por consenso entre os autores. Foi feito contato por e-mail com os pesquisadores dos estudos, quando necessário, para elucidar a elegibilidade de determinado estudo. Não foram impostas limitações de idiomas, datas ou status das publicações.

\subsubsection{Processo de coleta de dados}

Dois autores (CON, WPM), independentemente, extraíram os dados dos estudos incluídos utilizando um formulário piloto de extração de dados, planejado e testado por eles. Outro autor (DMT) conferiu os dados e discordâncias foram resolvidas por consenso. Os autores se corresponderam por e-mail com os pesquisadores dos estudos,quando necessário, para esclarecerem dúvidas sobre os dados. Os nomes dos autores dos artigos e títulos dos estudos incluídos foram 
sobrepostos procurando por publicações duplicadas. Essas publicações foram consideradas como parte de um mesmo estudo.

\subsection{Análise do risco de viés dos estudos incluídos}

Dois autores (CON e WPM) avaliaram, independentemente, os seguintes riscos de viés individualizados para cada estudo:

- Viés de seleção: ocultação da geração de sequência de randomização e alocação;

- Viés de performance: mascaramento de participantes e profissionais;

- Viés de detecção: mascaramento dos pesquisadores envolvidos na interpretação dos resultados;

- Viés de atrito: dados incompletos dos resultados se referem a pacientes que por algum motivo perderam o seguimento no decorrer do estudo;

- Viés de relato: relato seletivo de resultados;

- Outras fontes potenciais de viés: número de embriões transferidos.

Para a análise do risco de viés, foi utilizado o Cochrane Collaboration's criteria for judging risk of bias (Higgins; Green, 2011) e os estudos incluídos foram divididos em risco "baixo", "alto" ou "incerto" de viés.

\subsection{Medidas de efeito das intervenções}

O número de eventos no grupo controle (pacientes monitorizadas por meio de US associada à dosagem hormonal / US2D) e no grupo de intervenção (monitorização por US3D) de cada estudo foi usado para calcular o risco relativo (RR) de Mantel-haenszel. Optou-se pelo uso de RR, ao invés de odds ratio (OR), uma vez que o OR é de difícil compreensão e aplicação na prática. Há também a preocupação quanto à interpretação errônea do $\mathrm{OR}$, como se fosse equivalente 
ao $\mathrm{RR}$, isso tenderia hiperestimar o efeito da intervenção, em especial quando os eventos eram comuns (Higgins; Green, 2011). Porém, se fosse observada contagem de zero células ou prevalência <1, o OR Peto (modelo de efeito fixo) teria sido usado, pois esse método é menos sujeito aos riscos de viéses e mais eficaz, fornecendo maior cobertura do intervalo de confiança (IC) nessas situações. Adicionalmente, o valor do OR, nesses casos, é muito semelhante ao do RR, evitando-se erros de interpretação. A precisão das estimativas observadas foi avaliada para o IC de $95 \%$.

Foram considerados clinicamente relevantes quaisquer achados com significância estatística. Quando houve diferença estatística, o número necessário para produzir ou prevenir um desfecho foi calculado, ou número necessário para tratar (NNT) ou número necessário para causar dano (NNH), respectivamente.

\subsubsection{Unidades de análise}

Com o objetivo de analisar os dados de acordo com os princípios de intenção de tratamento, o número de mulheres alocadas foi usado como denominador, quando possível. Não foram encontrados estudos que incluíram os mesmos casais mais de uma vez (como estudos cross-over ou com dados "por ciclo"). A taxa de implantação não foi incluída na metanálise porque o denominador para esse desfecho (número de embriões transferidos) não foi randomizado; no entanto planejou-se incluir essa informação nas características dos estudos incluídos.

\subsubsection{Avaliação da heterogeneidade e avaliação da sensibilidade}

A heterogeneidade entre os estudos foi avaliada por meio do $\mathrm{I}^{2}$. Heterogeneidades importantes $\left(I^{2}>50 \%\right)$, entre os estudos, também foram analisadas. Primeiramente, quando confirmado que os dados foram corretamente extraídos, e se comprovada a heterogeneidade, utilizaram-se modelos de efeitos aleatórios para estimar o efeito da intervenção (uso apenas do US3D). Não foi realizada análise de subgrupos e a análise de sensibilidade foi aplicada para 
verificar se as conclusões relativas aos desfechos primários poderiam ser diferentes, caso a elegibilidade fosse restringida a estudos não classificados como de alto risco de viés. Interpretativamente, uma escala com valor de $l^{2}$ próximo a $0 \%$ indica não heterogeneidade entre os estudos, próximo a $25 \%$ sugere baixa heterogeneidade, próximo a $50 \%$, heterogeneidade moderada, e próximo a $75 \%$, alta heterogeneidade (Higgins; Thompson, 2002; Higgins et al. 2003; Rodrigues; Ziegelmann, 2010).

\subsubsection{Risco de viés entre os estudos}

Tendo em vista a dificuldade de se detectar e corrigir um possível viés de publicação, tentou-se minimizar o seu potencial impacto realizando busca apropriada de estudos elegíveis e permanecendo alerta à duplicação de dados publicados. Como foi pequeno o número de estudos incluídos, as avaliações de viés de publicação foi subótima, devido a impossibilidade de realização de análise do "gráfico de funil".

\subsection{Síntese de dados}

Os resultados foram combinados para metanálise com o Review Manager 5.1 (Copenhagen: The Nordic Cochrane Centre, The Cochrane Collaboration, 2011). A análise compara o uso de US associada à dosagem hormonal com US isolada, bem como o uso de US2D com US3D (incluindo sonoAVC) para proceder à monitorização do EOC. O aumento no risco de um desfecho particular associado à US isolada, que pode ser benéfico (ex.: nascidos vivos) ou prejudicial (ex.: SHO), foi apresentado na metanálise, graficamente à direita da linha central, enquanto a diminuição do risco de um desfecho aparece à esquerda da linha central. 


\subsection{Qualidade da evidência}

A qualidade da evidência para os principais resultados desta revisão foi avaliada conforme sugerido pelo Grading of Recommendations Assessment, Development and Evaluation (GRADE) working group (Guyatt et al., 2011), considerando os seguintes pontos: limitações dos estudos (ex.: risco de viés); inconsistência do efeito; imprecisão das estimativas; efeito indireto e viés de publicação. Julgamentos sobre a qualidade da evidência (alta, moderada, baixa) foram justificados, documentados e incorporados no relato dos resultados de cada desfecho avaliado (Guyatt et al., 2011). 
4. RESULTADOS 


\subsection{Resultados da seleção de estudos}

Foram selecionados 1717 registros: $C E N T R A L=445 ; C I N A H L=126$; Embase $=360 ;$ LILACS $=4 ;$ MEDLINE= 635; PsycINFO= 10; Clinical trials= 68; Controlled-trials= 28; WHO International Trials Registry Platform= 36. Cinco foram obtidos por busca manual, com base nas listas de referências dos estudos potencialmente elegíveis e revisões relacionadas. Após se removerem as duplicatas, 1320 registros foram rastreados com base nos títulos e resumos. Desses, 14 foram considerados potencialmente elegíveis e, posteriormente, avaliados quanto aos critérios de elegibilidade. Dos 14 estudos, quatro foram excluídos pelas seguintes razões: dois não eram randomizados (Kably Ambe et al., 1994; Murad, 1998), um era pseudo-randomizado (Murtinger et al., 2009) e um estava em andamento, na fase de recrutamento das participantes (Ben-Haroush, 2012). Dos 10 registros, nove foram incluídos na metanálise quantitativa (Figura 1).

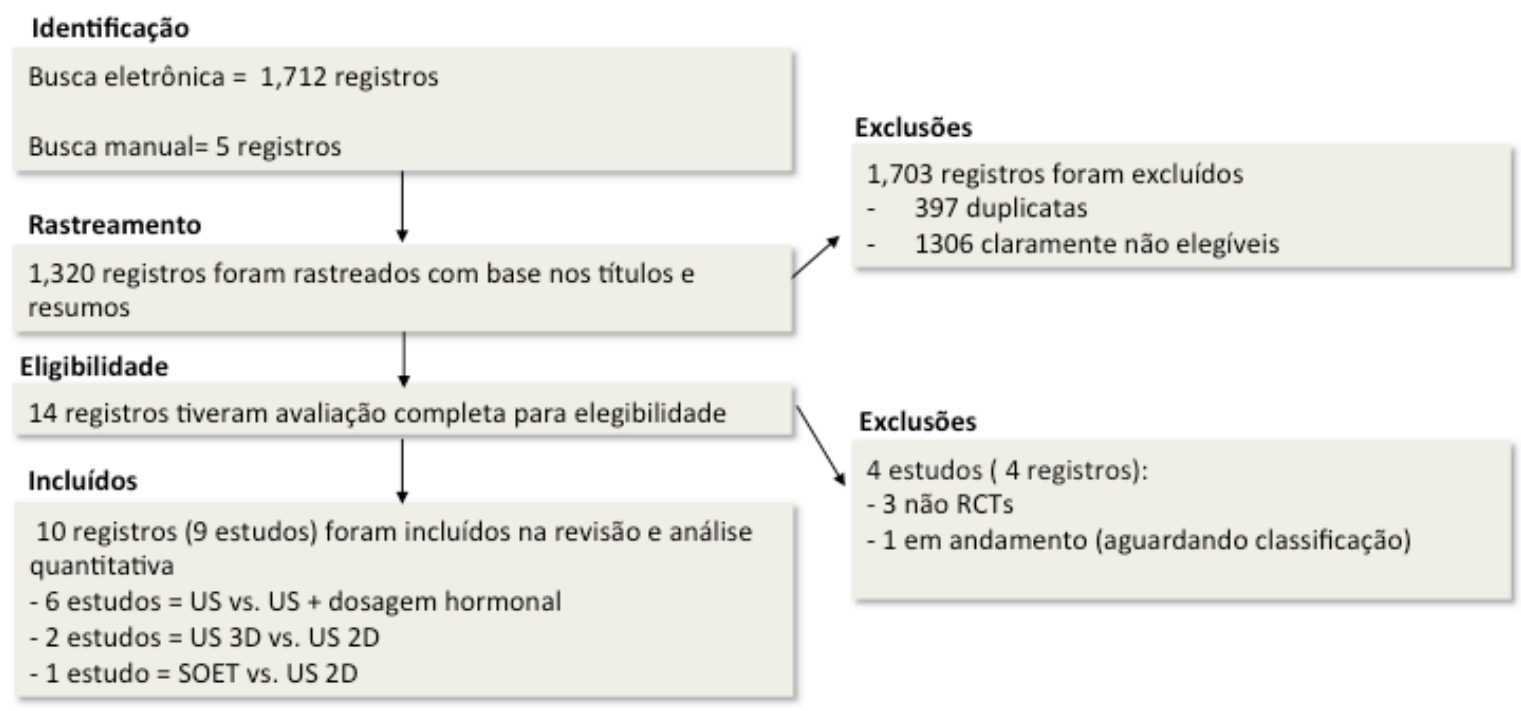

Figura 1: Fluxograma da seleção dos estudos. 


\subsection{Estudos Incluídos}

Todos os estudos incluídos eram RCTs. Oito eram unicêntricos conduzidos: três em Israel (Golan et al., 1994; Wiser et al., 2012; Nagar et al., 2014); um na Espanha (Aguirre et al., 2010), um no Reino Unido (Raine-Fenning et al., 2010), um na França (Rongieres, 2006), um nos Estados Unidos (Strawn et al., 2007) e um na Bélgica (Gerris et al., 2014). Outro estudo foi multicêntrico, envolvendo quatro centros de reprodução do Reino Unido (Lass, 2003), para este foi encontrado outro registro (Brinsden et al., 2001). Foi feito contato por e-mail com os autores de dois estudos para informações complementares (Lass, 2003; Wiser et al., 2012), sem obter resposta.

\subsection{Participantes}

Mil e trinta mulheres, dos nove estudos, foram incluídas: 393 foram avaliadas por US associada à dosagem hormonal, 513 somente por US2D, 63 realizaram monitorização do EOC por US3D e 61 SOET. Em um dos estudos foi permitido que as mulheres fizessem mais de um ciclo no mesmo grupo de alocação (Aguirre et al., 2010); 42 ciclos de 31 mulheres (média de 1.35 ciclos por mulher) em um grupo e 48 ciclos de 35 mulheres (média de 1.37 ciclos por mulher).

\subsection{Intervenções}

Seis (6/9) estudos compararam a monitorização do EOC por US isolada com a realizada por US associada à dosagem hormonal (Golan et al., 1994; Lass, 2003; Rongieres, 2006; Strawn et al., 2007; Aguirre et al., 2010; Wiser et al., 2012); dois (2/9) compararam a US3D com US2D para avaliação do EOC (RaineFenning et al., 2010; Nagar et al., 2014) e um estudo (1/9) comparou a SOET com a monitorização convencional por US2D (Gerris et al., 2014). Em três estudos 
(3/9), as mulheres monitoradas por US realizaram apenas a dosagem de E2 imediatamente antes da indução da maturação folicular final com hCG (Golan et al., 1994; Aguirre et al., 2010; Wiser et al., 2012).

\subsection{Resultados das análises dos estudos incluídos}

- Nenhum dos estudos reportou o número de nascidos vivos.

- Seis dos nove estudos reportaram número de casos de SHO (Golan et al., 1994; Lass, 2003; Rongieres, 2006; Strawn et al., 2007; Aguirre et al., 2010; Wiser et al., 2012).

- Seis dos nove estudos reportaram número de gravidez clínica (Lass, 2003; Rongieres, 2006; Strawn et al., 2007; Aguirre et al., 2010; RaineFenning et al., 2010; Wiser et al., 2012).

- Um dos nove estudos reportou abortamento (Aguirre et al., 2010).

- Oito dos nove estudos reportaram número total de oócitos captados (Golan et al., 1994; Lass, 2003; Strawn et al., 2007; Aguirre et al., 2010; Raine-Fenning et al., 2010; Wiser et al., 2012; Gerris et al., 2014; Nagar et al., 2014).

- Um dos nove estudos reportou taxa de implantação (Aguirre et al., 2010).

\subsection{Estudos excluídos}

Três estudos foram excluídos da revisão, pelas seguintes razões: dois que comparavam a monitorização do EOC por US isolada com US associada à dosagem hormonal, não eram randomizados (Kably Ambe et al., 1995; Murad, 1998) e outro, que comparava a monitorização do EOC por US3D com US2D, tratava-se de um estudo pseudo-randomizado (Murtinger et al., 2009) (Tabela 1). 
Tabela 1 - Característica dos estudos excluídos.

\begin{tabular}{ll}
\hline \multicolumn{1}{c}{ Estudo } & \multicolumn{1}{c}{ Razão da Exclusão } \\
\hline (Kably et al. 1994) & Estudo não randomizado \\
(Murad et al. 1998) & Estudo não randomizado \\
(Murtinger et al. 2009) & Estudo pseudo-randomizado \\
\hline
\end{tabular}

\subsection{Estudos aguardando classificação}

Um estudo foi localizado por meio de busca eletrônica em 15 de abril de 2013 e novamente em 12 de março de 2015, considerado potencialmente elegível, com base na leitura do título e resumo (Ben-Haroush, 2012). O estudo está em andamento, ainda sem publicação disponível. Tentou-se contato via email com o autor, sem resposta. Até a data de finalização deste estudo, não foi possível obter informações suficientes para julgá-lo elegível. Por esse motivo foi considerado "aguardando classificação".

\subsection{Risco de viés nos estudos incluídos}

Seis estudos aplicaram adequadamente os métodos de randomização (Lass, 2003; Rongieres, 2006; Aguirre et al., 2010; Raine-Fenning et al., 2010; Wiser et al., 2012; Gerris et al., 2014); em três, o método de randomização não foi descrito (Golan et al., 1994; Strawn et al., 2007; Nagar et al., 2014) e sete foram julgados como de risco de viés incerto, porque o método de ocultação da randomização não foi descrito claramente (Golan et al., 1994; Rongieres, 2006; Strawn et al., 2007; Aguirre et al., 2010; Raine-Fenning et al., 2010; Gerris et al., 2014; Nagar et al., 2014). Não se considerou que o mascaramento dos profissionais e participantes pudesse influenciar o risco de viés de performance desta revisão. Três estudos foram julgados de alto risco de viés de atrito, porque em dois deles algumas participantes foram excluídas da análise (Aguirre et al., 
2010; Wiser et al., 2012), sendo que em um estudo (Aguirre et al., 2010), quatro mulheres foram excluídas da análise porque não seguiram o protocolo estabelecido e no outro (Wiser et al., 2012), duas foram excluídas após alocação, pois uma delas ficou grávida antes do EOC e a outra deixou a clínica antes de iniciar o tratamento. No terceiro (Gerris et al., 2014), duas participantes que estavam no braço SOET trocaram para monitorização tradicional devido à falha técnica do probe, mas no princípio (intention to treatment - ITT) foram analisadas no grupo SOET. Dois estudos estavam sob risco de viés de atrito incerto, por não mencionarem o seguimento das pacientes (Strawn et al., 2007; Nagar et al., 2014). Um estudo foi considerado sob alto risco de viés de relato (Golan et al., 1994), pois os autores descreveram a proporção de gravidez clínica por oócito captado e embrião transferido, porém não reportaram o número de mulheres submetidas à captação de oócitos e/ou transferência de embriões em cada grupo. Consequentemente, não foi possível determinar o número total de gravidez clínica em cada grupo. Outro estudo (Lass, 2003) estava sob alto risco de outros viéses, pois a intervenção inicial proposta para o grupo US associada à dosagem hormonal foi modificada durante o ensaio: 40 de 148 mulheres não atingiram o critério, inicialmente proposto, para administração do hCG e receberam a medicação mesmo assim, seguindo o critério sugerido para o grupo de monitorização do EOC apenas por US. Não foram observadas diferenças significativas nos estudos entre os grupos ao reportarem média de idade, IMC ou $\mathrm{FSH}$.

\subsection{Síntese de resultados}

\subsubsection{Comparação US isolado vs. US + dosagem hormonal}

\subsubsection{Nascimento vivo}

Nenhum estudo reportou esse desfecho. 


\subsubsection{SHO}

O IC observado foi bastante amplo e não permitiu concluir pela existência de benefício nem prejuízo para a intervenção com US isolada para monitorização do EOC, comparada à US associada à dosagem hormonal (OR=1.03, IC95\% 0.48 a $2.18, p=0.95$ ), seis estudos, 774 mulheres, $I^{2}=0$, evidência de baixa qualidade (Figura 2). A análise de sensibilidade, restrita aos estudos julgados sem alto risco de viés, não mudou a estimativa de efeito da intervenção (OR=0.78, IC95\% 0.27 a 2.28, $\mathrm{p}=0.65)$, dois estudos, 299 mulheres.

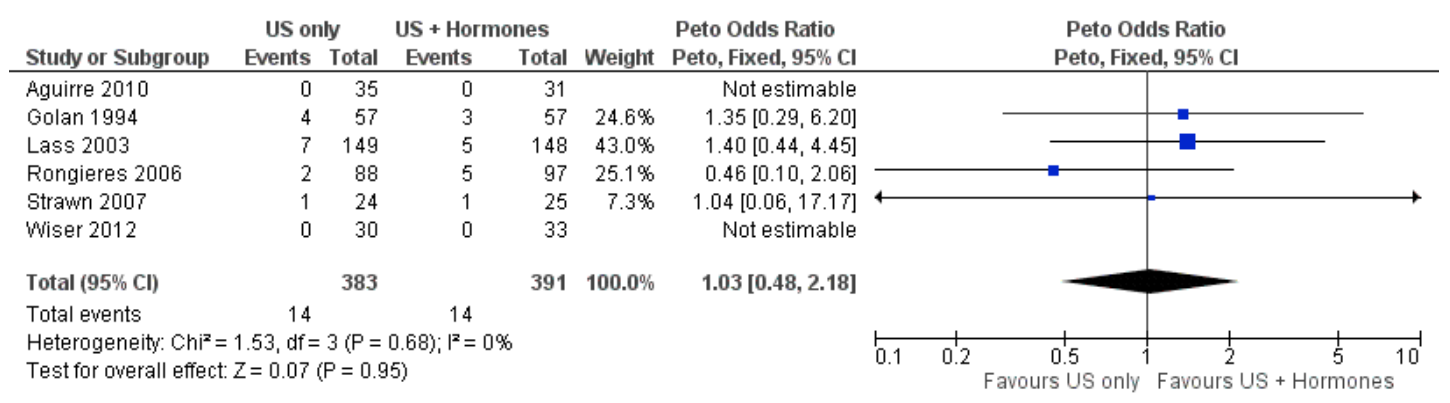

Figura 2: Síndrome de hiperestímulo ovariano (SHO)- Forest Plot: Comparação US Isolada versus US + dosagem Hormonal.

\subsubsection{Gravidez clínica}

O IC observado foi amplo e não permitiu concluir pela existência de benefício nem prejuízo para a intervenção US isolada para monitorização do EOC comparada à US associada à dosagem hormonal $(\mathrm{RR}=0.96, \mathrm{IC} 95 \% 0.80$ a 1.16 , $p=0.70$ ), cinco estudos, 660 mulheres, $\left.\right|^{2}=10 \%$, evidência de baixa qualidade (Figura 3). Na análise de sensibilidade, restrita aos estudos julgados sem alto risco de viés, o IC foi muito extenso e não permitiu concluir pela existência de benefício nem prejuízo para a intervenção US isolada comparada à US associada à dosagem hormonal para a monitorização do $E O C(R R=0.96, C 195 \% 0.62$ a 1.48, $p=0.84$ ), um estudo, 185 mulheres. 


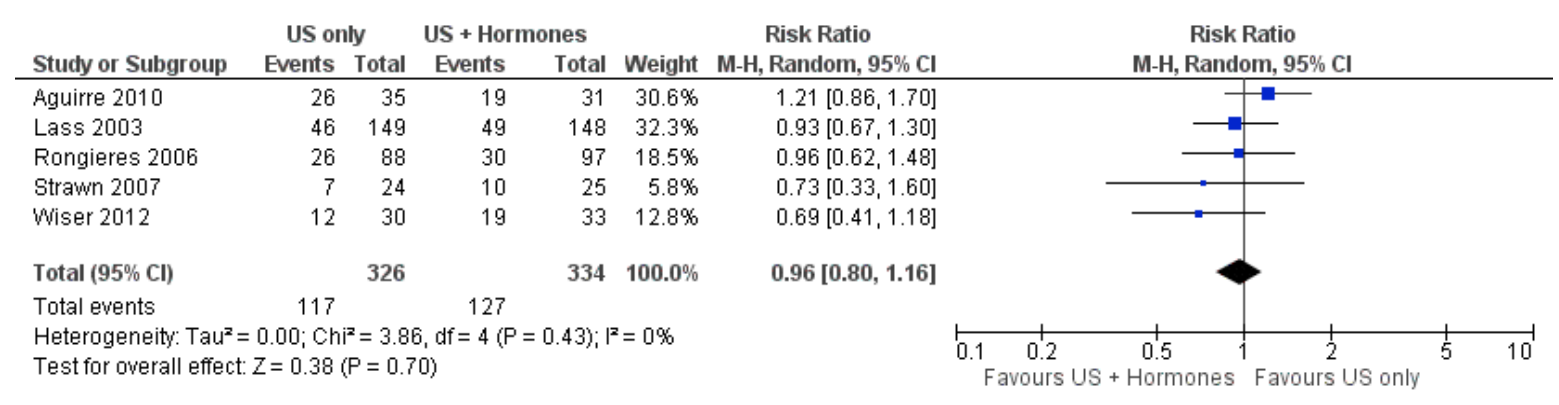

Figura 3: Gravidez clínica - Forest Plot: comparação US Isolada versus US + dosagem Hormonal.

\subsubsection{Abortamento por gravidez clínica}

Apenas um estudo relatou o desfecho abortamento e o IC era muito amplo. Os achados não permitiram concluir pela existência de benefício nem prejuízo para a intervenção US isolada comparada à US associada à dosagem hormonal para monitorização do EOC ( $R R=0.37, \mathrm{IC} 95 \% 0.07$ a 1.79, $\mathrm{p}=0.21)$, um estudo, 45 mulheres, evidência de muito baixa qualidade (Figura 4). A análise de sensibilidade não foi realizada, pois o único estudo que reportou aborto foi considerado de alto risco de viés.

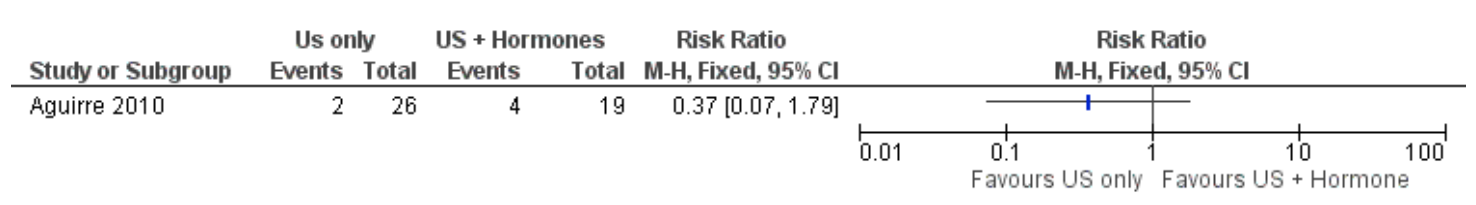

Figura 4: Abortamento por gravidez clínica - Forest Plot: comparação US Isolada versus US + dosagem Hormonal.

\subsubsection{Número total de oócitos captados}

O IC observado foi amplo, compatível com benefício apreciável ou nenhum efeito para a intervenção US isolada comparada à US associada à dosagem hormonal para monitorização do EOC (Diferença média - MD=0.92 oócitos captados, $\mathrm{Cl} 95 \%-0.19$ a $2.04, \mathrm{p}=0.70$ ), quatro estudos, 523 mulheres, $\mathrm{I}^{2}=0$, 
moderada qualidade de evidência (Figura 5). Quando realizada a análise de sensibilidade, o IC foi amplo e compatível com benefício apreciável, sem efeito, ou pequeno prejuízo para o uso de US isolada comparada à US associada à dosagem hormonal: (MD=1.70 oócitos captados, IC95\% -1.22 a 4.62, p=0.25), um estudo, 114 mulheres.

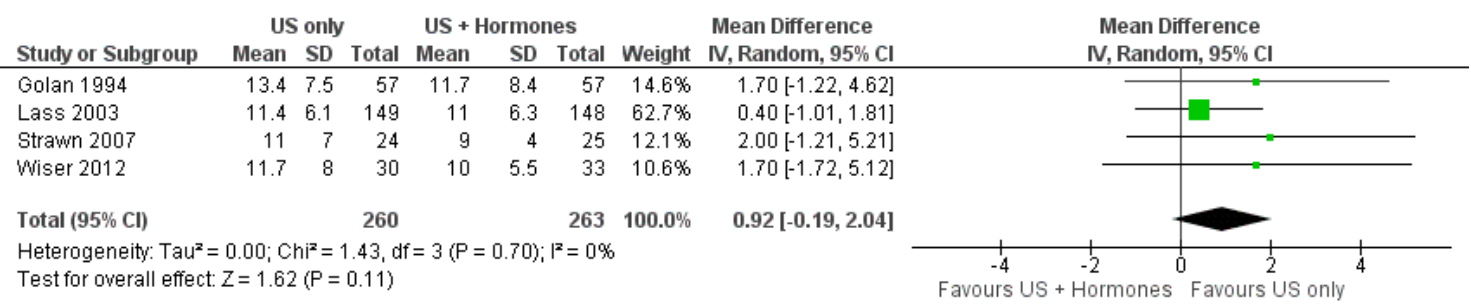

Figura 5: Número de oócitos captados- Forest Plot: comparação US Isolada versus US + dosagem Hormonal.

\subsubsection{Comparação US3D vs. US2D}

Dois estudos foram incluídos nessa comparação (Raine-Fenning et al., 2010; Nagar et al., 2014), e esses não reportaram nascidos vivos, SHO ou abortamento. Os estudos foram julgados como não sendo de alto risco de viés e não foram identificadas evidências de viés de publicação ou de seleção.

\subsubsection{Gravidez clínica}

Quando o desfecho avaliado foi gravidez clínica, um dos estudos (Nagar et al., 2014) reportou não ter encontrado diferenças estatisticamente significativas entre monitorar o EOC por meio de US3D ou US2D, porém não forneceu dados numéricos. Outro estudo (Raine-Fenning et al., 2010) forneceu dados para a análise e o IC foi extenso e não permitiu concluir por benefício nem prejuízo associado a intervenção US3D comparada à US2D para monitorização do EOC $(R R=1, C \mid 95 \% 0.58$ a 1.73, $p=1.00)$, um estudo, 72 mulheres (Figura 6). 


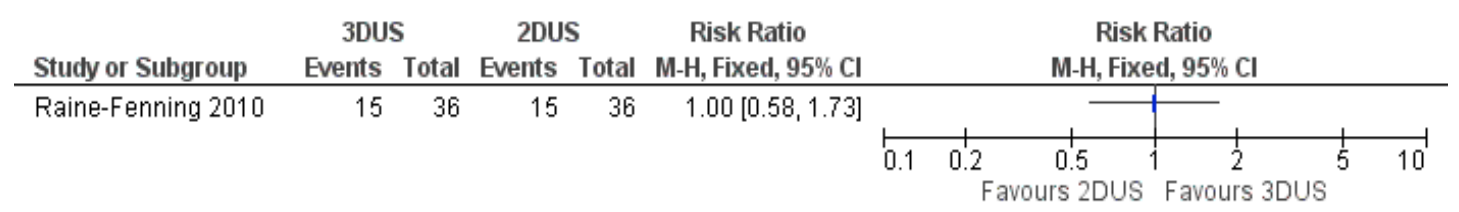

Figura 6: Gravidez clínica - Forest Plot: comparação US3D versus US2D.

\subsubsection{Número total de oócitos captados}

Os dois estudos incluídos na comparação entre o uso de US3D e de US2D para monitorização do EOC relataram o número total de oócitos captados. Um deles (Nagar et al., 2014) reportou não ter encontrado diferenças estatisticamente significativas relacionadas ao número de oócitos captados entre monitorar o EOC por meio de US3D ou US2D, porém não forneceu dados numéricos. O estudo de Raine-Fenning et al. (2010) forneceu dados para a análise e o IC foi amplo e compatível com benefício apreciável, sem efeito, prejuízo considerável para a intervenção uso de US3D comparado ao uso de US2D para monitorização do EOC (MD= -037, CI95\% -3.63 a 2.89, p=0.82), um estudo, 72 mulheres (Figura 7).

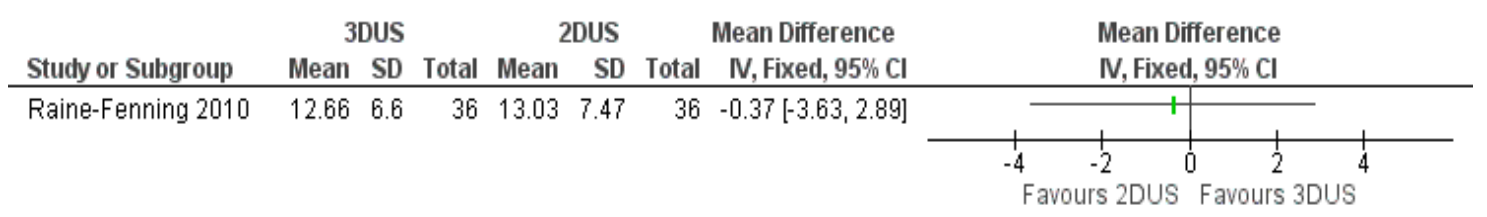

Figura 7: Número total de oócitos captados - Forest Plot: comparação US3D versus US2D.

\subsubsection{Comparação SOET vs.US 2D}

Apenas um estudo foi incluído nessa comparação, e este não reportou nascidos vivos, SHO ou abortamento. Os autores avaliaram gravidez clínica e número total de oócitos captados (Gerris et al., 2014). 


\subsubsection{Gravidez clínica}

O IC observado foi amplo e não permitiu concluir por benefício nem prejuízo associado a intervenção uso de US2D para monitorização do EOC comparada à SOET (RR=0.95, IC95\% 0.52 a 1.75, $\mathrm{p}=0.88)$, um estudo, 123 mulheres, evidência de baixa qualidade (Figura 8).

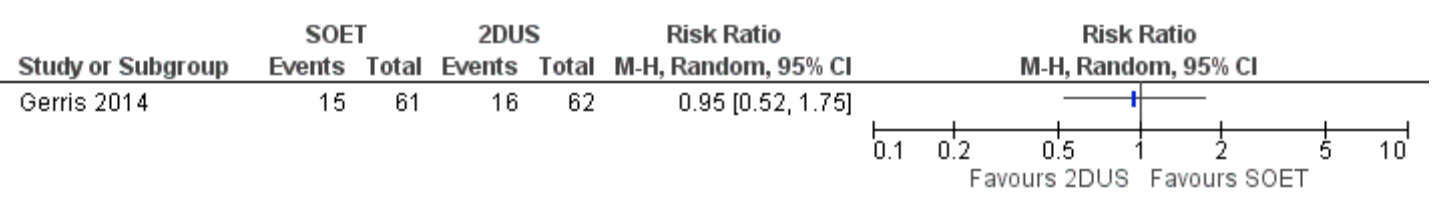

Figura 8: Gravidez clínica - Forest Plot: comparação SOET versus US2D.

\subsubsection{Número total de oócitos captados}

Gerris et al. (2014) forneceram dados para a análise e o IC foi amplo e não permitiu concluir pela existência de benefício nem prejuízo para a intervenção com SOET comparada à US2D para monitorização do EOC (MD=0.50, C195\% 2.13 a $3.13, p=0.71$ ), um estudo, 123 mulheres (Figura 9).

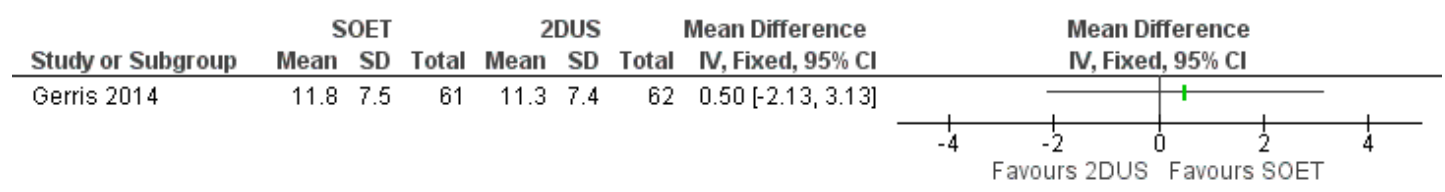

Figura 9: Número total de oócitos captados - Forest Plot: comparação SOET versus US2D. 
5. DISCUSSÃO 


\subsection{Síntese dos principais resultados}

Seis estudos foram incluídos na comparação US isolada versus US associada à dosagem hormonal. Nenhum deles avaliou nascimentos vivos (Tabela 2).

Tabela 2- Detalhes dos estudos incluídos na comparação US + E2 vs. US.

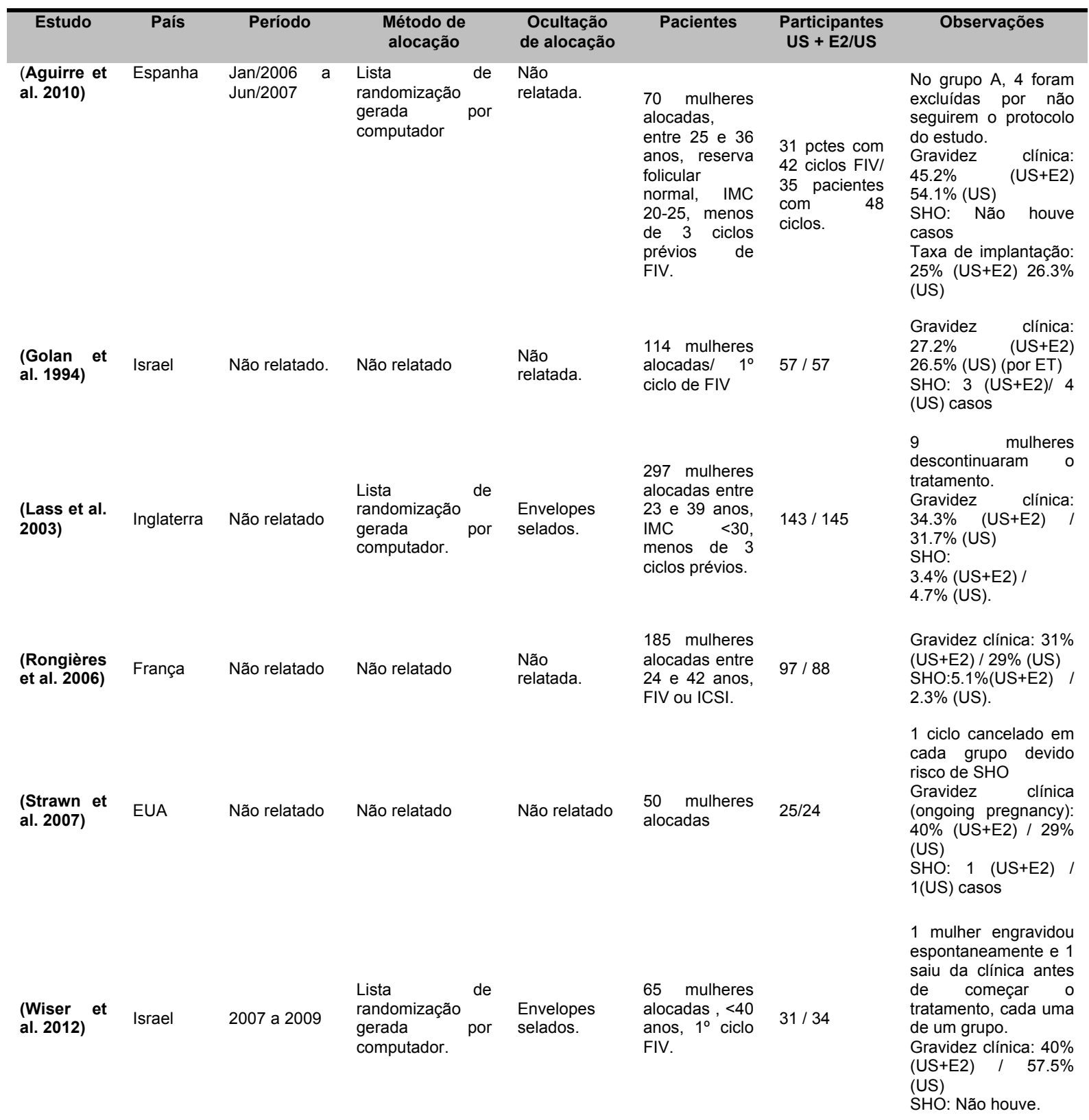


Observou-se que monitorar o EOC apenas com US parece não causar redução do número de oócitos captados quando comparada com a monitorização por US associada à dosagem hormonal. Essa evidência foi julgada de moderada qualidade, o que não permitiu concluir a superioridade do método utilizado como padrão em relação à US apenas. Também notou-se que monitorar o EOC com US isolado não muda substancialmente a chance de se alcançar gravidez clínica, essa evidência foi considerada de baixa qualidade, o que causa incerteza quanto ao seu efeito. O mesmo se aplica para SHO.

Como apenas um estudo (Aguirre et al., 2010) reportou uso de US isolada versus US + dosagem hormonal para o desfecho abortamento e este foi julgado como de evidência muito baixa, não foi possível concluir o efeito da intervenção sobre esse desfecho. Apenas dois estudos incluídos (Raine-Fenning et al., 2010; Nagar et al., 2014) compararam o uso de US3D versus US2D para monitorar a ovulação e um deles (Raine-Fenning et al., 2010) forneceu evidências sobre o número de oócitos captados e gravidez clínica. No entanto, as estimativas não foram suficientemente precisas para se concluir pela existência de benefício nem prejuízo. Nagar et al. (2014) não encontraram diferenças significativas entre a monitorização realizada por US3D e US2D em relação ao número total de oócitos captados, número de oócitos maduros e porcentagem de oócitos maduros, taxas de gravidez ou de implantação. Dessa forma, também não foi possível concluir se houve vantagem estatisticamente significativa no uso de US3D (Tabela 3).

Tabela 3- Detalhes dos estudos incluídos na comparação US2D vs. US3D.

\begin{tabular}{|c|c|c|c|c|c|c|c|}
\hline Estudo & País & Período & $\begin{array}{l}\text { Método de } \\
\text { alocação }\end{array}$ & $\begin{array}{l}\text { Ocultação } \\
\text { de alocação }\end{array}$ & Pacientes & $\begin{array}{l}\text { Participantes } \\
\text { US2D/US3D }\end{array}$ & Observações \\
\hline $\begin{array}{l}\text { (Raine } \\
\text { Fenning et } \\
\text { al. 2010) }\end{array}$ & Inglaterra & $\begin{array}{l}\text { Jan2006 a } \\
\text { jun/2007 }\end{array}$ & $\begin{array}{l}\text { Lista de } \\
\text { randomização } \\
\text { gerada por } \\
\text { computador. }\end{array}$ & Não relatado & $\begin{array}{lr}72 & \text { mulheres } \\
\text { alocadas, } & \text { idade } \\
<38 \text { anos, IMC }<28, \\
\text { reserva } \quad \text { ovariana } \\
\text { normal }(\mathrm{FSH}<10 \mathrm{IU}) .\end{array}$ & $34 / 34$ & $\begin{array}{l}4 \text { pctes foram } \\
\text { excluídas. } \\
\text { Gravidez clínica: } \\
42 \% \text { (US2D) / } \\
43 \% \text { (US3D) }\end{array}$ \\
\hline $\begin{array}{l}\text { (Nagar et } \\
\text { al. 2014)) }\end{array}$ & Israel & $\begin{array}{c}\text { Não } \\
\text { relatado }\end{array}$ & Não relatado & Não relatado & 54 mulheres & $27 / 27$ & \\
\hline
\end{tabular}

Um estudo incluído comparou a SOET com a monitorização convencional da ovulação por US2D (Gerris et al., 2014) e forneceu evidências sobre o número 
de oócitos captados e gravidez clínica, no entanto as estimativas não foram suficientemente precisas para se concluir benefício ou prejuízo (Tabela 4).

Tabela 4 - Detalhes do estudo incluído na comparação US2D vs. SOET.

\begin{tabular}{|c|c|c|c|c|c|c|c|}
\hline Estudo & País & Período & $\begin{array}{l}\text { Método de } \\
\text { alocação }\end{array}$ & $\begin{array}{c}\text { Ocultação } \\
\text { de alocação }\end{array}$ & Pacientes & $\begin{array}{l}\text { Participantes } \\
\text { US2D/SOET }\end{array}$ & Observações \\
\hline $\begin{array}{l}\text { (Guerris et } \\
\text { al. 2014) }\end{array}$ & Bélgica & $\begin{array}{l}\text { Não } \\
\text { relatado }\end{array}$ & Não relatado & Não relatado & $\begin{array}{l}123 \text { mulheres alocadas, } \\
\text { idade <41 anos, ICSI, } \\
\text { presença dos dois ovários, } \\
\text { excluídas mas } \\
\text { respondedoras }\end{array}$ & $59 / 62$ & \\
\hline
\end{tabular}

ICSI= Injeção intracitoplasmática de espermatozoide; US2D= Ultrassonografia em duas dimensões; SOET= telemonitorização endovaginal operada pela própria paciente.

\subsection{Qualidade das evidências}

$\mathrm{Na}$ comparação US isolada vs. US + dosagem hormonal para monitorização do EOC (Tabela 2), as evidências produzidas pela metanálise foram consideradas de baixa qualidade. Os IC amplos e o alto risco de viés dos estudos incluídos contribuíram para o rebaixamento da qualidade das evidências. Para a SHO, a qualidade de evidência foi considerada baixa: rebaixada um nível devido à imprecisão (IC95\% amplo) e outro nível porque foi observado alto risco de viés nos estudos que avaliaram tal desfecho. A qualidade de evidência para gravidez clínica também foi considerada baixa: rebaixada um nível devido imprecisão e mais um nível pelo alto risco de viés dos estudos incluídos.

$\mathrm{Na}$ avaliação de abortamento, a qualidade de evidência foi julgada muito baixa: rebaixada dois níveis devido séria imprecisão (IC95\% muito amplo) e outro nível devido alto risco de viés. A qualidade de evidência para número de oócitos captados foi considerada moderada: rebaixada um nível devido ao alto risco de viés dos estudos.

Na comparação US3D versus US2D (Tabela 3), a qualidade de evidência para os dois desfechos reportados (gestação clínica e número de oócitos captados) foi considerada baixa: rebaixada dois níveis devido à séria imprecisão (IC95\% muito amplo), nos dois estudos incluídos. Na comparação SOET versus monitorização convencional por US2D (Tabela 4), a qualidade de evidência para 
número de oócitos captados e gravidez clínica (ongoing pregnancies) foi julgada baixa.

\subsection{Completude geral e aplicabilidade da evidência}

Outra revisão sistemática, que contempla este assunto, foi publicada em 2008 (Kwan et al., 2008). Nela, apenas dois estudos foram incluídos. Revisada em 2014, foram adicionados quatro estudos (Kwan et al., 2014). Os autores inferiram que as evidências eram muito escassas até aquele ponto para gerar alguma conclusão. Foram incluídos mais quatro estudos: um de 2006 (Rongieres, 2006) e outros três (Aguirre et al., 2010; Raine-Fenning et al., 2010; Wiser e al., 2012).

Além dos seis estudos incluídos na análise quantitativa, três outros abordavam o tema desta revisão, mas foram excluídos por não serem randomizados (Kably Ambe et al., 1994; Murad, 1998; Murtinger et al., 2009). Dois desses ensaios (Kably Ambe et al., 1994; Murad, 1998) avaliaram a comparação US isolada versus US + dosagem hormonal. Um deles (Murad, 1998) reportou nascidos vivos ( $R R=1.05, I C 95 \% 0.47$ a 2.32, $p=0.91,206$ mulheres); SHO (OR=0.87 IC95\% 0.05 a 14.12, p=0.92, 206 mulheres) e gestação clínica por mulher alocada ( $R R=1.04, I C 95 \% 0.57$ a 1.90, $p=0.91,206$ mulheres). Outro estudo, (Kably Ambe et al., 1994) relatou gestação clínica por mulher alocada ( $R R=0.83, I C 95 \% 0.25$ a 2.80, $p=0.77,120$ mulheres) e número de oócitos captados $(\mathrm{MD}=-0.30, \mathrm{IC95} \%-1.63$ a 1.03, $\mathrm{p}=0.66,120$ mulheres). O terceiro (Murtinger et al., 2009) avaliou o uso do sonoAVC na comparação entre US3D e US2D e mostrou: gravidez clínica por mulher alocada $(R R=0.90, I C 95 \% 0.47$ a 1.73, $p=0.75,40$ mulheres); número de oócito captados ( $\mathrm{MD}=3.20$ oócitos, $\mathrm{C} 195 \%$ -0.89 a 7.29, $p=0.12,40$ mulheres). Nenhum dos três estudos observou diferença significativa entre os grupos comparados, o que corrobora os achados dos estudos incluídos nesta metanálise.

Monitorar o EOC com US associada à dosagem hormonal aumenta os custos e é causa de desconforto e estresse relacionados ao procedimento de 
coleta de amostras de sangue da paciente. Todavia, não há certeza dos efeitos dessa intervenção relacionados a desfechos importantes como taxa de gestação clínica e risco de SHO. Monitorar o EOC somente por US não parece reduzir o número de oócitos captados ou causar mudanças substanciais nas chances de se alcançar gravidez clínica. Assim sendo, acredita-se que a monitorização do EOC, apenas por US, deva ser considerada como alternativa sensata.

\subsection{Limitações}

Embora os estudos incluídos fossem considerados consistentes na estimativa de efeito, a qualidade da evidência verificada nesta revisão foi comprometida pela qualidade desses estudos, pois três de seis estudos foram julgados estarem sob alto risco de viés. Apesar de as razões principais em eliminar as dosagens hormonais e incluir as técnicas semiautomáticas de medidas ultrassonográficas serem a redução dos custos e do estresse, além da melhora da logística, nenhum dos estudos incluídos avaliou o custo-efetividade e/ou a qualidade de vida relacionada à monitorização do EOC. Apenas um estudo, que foi excluído por não ser randomizado (Murad, 1998), avaliou o custo da monitoração por US isolada vs. US associada à dosagem hormonal e encontrou redução de $43 \%$ em benefício do primeiro.

O pequeno número de RCTs fez com que a avaliação dos viéses de publicação e relato fosse subótima devido a impossibilidade de se realizar a análise de gráfico de funil. 
6. CONCLUSÕES 
A monitorização do EOC apenas por US é possível e não parece causar redução relevante no número de oócitos captados nem mudanças substanciais nas chances de se atingir a gestação clínica quando comparada à realizada por meio de US associada à dosagem hormonal. Entretanto, ainda há incerteza dos efeitos sobre outros desfechos relevantes como taxa de nascidos vivos, SHO e abortamento. Também não há segurança quanto ao efeito do uso de US3D para a monitorização do EOC. Assim, considera-se que será necessário maior número de estudos que avaliem a melhor forma de monitorização do EOC.

Este estudo concorda com as revisões de Kwan et al. $(2008,2014)$ quanto à dificuldade em se realizar um RCT com tamanho amostral suficiente para que sejam testados os efeitos dos diferentes protocolos de monitorização sobre um desfecho tão raro quanto a SHO. Porém, devido a isso, porque monitorar com E2 também? Os critérios US não seriam suficientes? E alguns estudos ainda relatam a não correlação ou dissociação entre os níveis de E2 durante a administração exógena de $\mathrm{FSH}$, durante o EOC.

\section{Implicações para pesquisa}

Serão necessários mais ensaios clínicos randomizados, com maior tamanho amostral, para a avaliação do efeito da monitorização do EOC por US isolada sobre nascidos vivos, gravidez clínica, SHO, número de oócitos captados, abortamento, bem como a repercussão socioeconômica. 


\section{REFERÊNCIAS BIBLIOGRÁFICAS ${ }^{1}$}

1Elaborada de acordo com a Associação Brasileira de Normas Técnicas. NBR 6023. 
Abdalla HI, Baber RJ, Leonard T, Kirkland A, Mitchell A, Power M, et al. Timed oocyte collection in an assisted conception programme using $\mathrm{GnRH}$ analogue. Hum Reprod. 1989;4(8):927-30.

Aguirre SG, Hevia IG, Garcia-Carpintero G, Del Rosal Ma J. Measuring plasma estradiol levels in in vitro fertilization. Is it useful? Rev Iberoamer Fertil Reprod Hum. 2010;27(1):43-9.

Ben-Haroush A. Automated 3D (SonoAVC) Versus 2D Ovarian Follicles Assessment in IVF Cycles: a Randomized Controlled Trial. NCT01723267. 2012. Disponível em: https://clinicaltrials.gov/ct2/show/NCT01723267. Acessado em 15 nov 2014.

Brinsden P, Abdalla S, Barlow D, Goswamy R, Maislish L, Larroque S, et al. A prospective, multicentre, randomized, patient-blind, parallel-group study comparing ultrasound only criterion for administration of UHCG with an oestradiol/follicle ratio criterion in down-regulated patients treated with rHFSH prior to IVF-ET. Human Reprod. 2001;16(Suppl 1):72.

Cantwell R, Clutton-Brock T, Cooper G, Dawson A, Drife J, Garrod D, et al. Saving Mothers' Lives: Reviewing maternal deaths to make motherhood safer: 2006-2008. The Eighth Report of the Confidential Enquiries into Maternal Deaths in the United Kingdom. BJOG. 2011;118(Suppl 1):1-203.

Deutch TD, Joergner I, Matson DO, Oehninger S, Bocca S, Hoenigmann D, et al. Automated assessment of ovarian follicles using a novel three-dimensional ultrasound software. Fertil Steril. 2009;92(5):1562-8.

Evers JL. Female subfertility. Lancet. 2002;360(9327):151-9.

Gerris J, De Sutter P. Self-operated endovaginal telemonitoring (SOET): a step towards more patient-centred ART? Hum Reprod. 2010;25(3):562-8.

Gerris J, Delvigne A, Dhont N, Vandekerckhove F, Madoc B, Buyle M, et al. Selfoperated endovaginal telemonitoring versus traditional monitoring of ovarian stimulation in assisted reproduction: an RCT. Hum Reprod. 2014;29(9):1941-8.

Gnoth C, Godehardt E, Frank-Herrmann P, Friol K, Tigges J, Freundl G. Definition and prevalence of subfertility and infertility. Hum Reprod. 2005;20(5):1144-7. 
Gnoth C, Maxrath B, Skonieczny T, Friol K, Godehardt E, Tigges J. Final ART success rates: a 10 years survey. Hum Reprod. 2011;26(8):2239-46.

Golan A, Herman A, Soffer Y, Bukovsky I, Ron-El R. Ultrasonic control without hormone determination for ovulation induction in in-vitro fertilization/embryo transfer with gonadotrophin-releasing hormone analogue and human menopausal gonadotrophin. Hum Reprod. 1994;9(9):1631-3.

Guyatt G, Oxman AD, Akl EA, Kunz R, Vist G, Brozek J, et al. GRADE guidelines: 1. Introduction-GRADE evidence profiles and summary of findings tables. J Clin Epidemiol. 2011;64(4):383-94.

Higgins JP, Thompson SG. Quantifying heterogeneity in a meta-analysis. Stat Med. 2002;21(11):1539-58.

Higgins JPT, Thompson SG, Deeks JJ, Altman DG. Measuring inconsistency in meta-analysis. BMJ. 2003;327:527-60.

Higgins J, Green S. (editors) Cochrane Handbook for Systematic Reviews of Interventions Version 5.1.0 [updated March 2011]: The Cochrane Collaboration. Disponível em: http://www.cochrane-handbook.org. Acessado 15 nov 2014.

Humaidan P, Papanikolaou EG, Kyrou D, Alsbjerg B, Polyzos NP, Devroey P, et al. The luteal phase after $\mathrm{GnRH}$-agonist triggering of ovulation: present and future perspectives. Reprod Biomed Online. 2012;24(2):134-41.

Kably Ambe A, Carranza Lira S, Serviere Zaragoza C, Espinoza de los Monteros A, Coria Soto I, Alvarado Duran A. The evaluation of the usefulness of ultrasound independent of estradiol as a prognostic criterion for ovular capture and maturity. A comparative double-blind study. Ginecol Obstet Mex. 1994;62:288-91.

Kwan I, Bhattacharya S, McNeil A, van Rumste MM. Monitoring of stimulated cycles in assisted reproduction (IVF and ICSI). Cochrane Database Syst Rev. 2008;2:CD005289.

Kwan I, Bhattacharya S, Kang A, Woolner A. Monitoring of stimulated cycles in assisted reproduction (IVF and ICSI). Cochrane Database Syst Rev. 2014;8:CD005289. 
Kyei-Mensah A, Zaidi J, Pittrof R, Shaker A, Campbell S, Tan SL. Transvaginal three-dimensional ultrasound: accuracy of follicular volume measurements. Fertil Steril. 1996;65(2):371-6.

Lass A. Monitoring of in vitro fertilization-embryo transfer cycles by ultrasound versus by ultrasound and hormonal levels: a prospective, multicenter, randomized study. Fertil Steril. 2003;80(1):80-5.

Macklon NS, Fauser BC. Mild stimulation in in vitro fertilization. Ann N Y Acad Sci. 2003;997:105-11.

Macklon NS, Stouffer RL, Giudice LC, Fauser BC. The science behind 25 years of ovarian stimulation for in vitro fertilization. Endocr Rev. 2006;27(2):170-207.

Martins WP, Rocha IA, Ferriani RA, Nastri CO. Assisted hatching of human embryos: a systematic review and meta-analysis of randomized controlled trials. Hum Reprod Update. 2011;17(4):438-53.

Murad NM. Ultrasound or ultrasound and hormonal determinations for in vitro fertilization monitoring. Int J Gynaecol Obstet. 1998;63(3):271-6.

Murtinger $\mathrm{M}$, Aburumieh $\mathrm{A}$, Rubner $\mathrm{P}$, Eichel V, Zech $\mathrm{MH}$, Zech $\mathrm{NH}$. Improved monitoring of ovarian stimulation using $3 \mathrm{D}$ transvaginal ultrasound plus automated volume count. Reprod Biomed Online. 2009;19(5):695-9.

Nagar R, Tenenbaum-Gavish K, Fisch B, Meizner I, Ben-Haroush A. OC22.07: Fertility treatment outcomes following standard 2D ultrasound vs 3D automated follicle tracking (sonoAVC). Ultrasound Obstet Gynecol. 2014;44(S1):52-3.

Nastri CO, Ferriani RA, Rocha IA, Martins WP. Ovarian hyperstimulation syndrome: pathophysiology and prevention. J Assist Reprod Genet. 2010;27(23):121-8.

Nastri CO, Gibreel A, Raine-Fenning N, Maheshwari A, Ferriani RA, Bhattacharya $S$, et al. Endometrial injury in women undergoing assisted reproductive techniques. Cochrane Database Syst Rev. 2012;7:CD009517. 
Nastri CO, Teixeira DM, Moroni RM, Leitao VM, Martins WP. Ovarian hyperstimulation syndrome: pathophysiology, staging, prediction and prevention. Ultrasound Obstet Gynecol. 2015;45(4):377-93.

Pelinck MJ, Vogel NE, Hoek A, Simons AH, Arts EG, Mochtar MH, et al. Cumulative pregnancy rates after three cycles of minimal stimulation IVF and results according to subfertility diagnosis: a multicentre cohort study. Hum Reprod. 2006;21(9):2375-83.

Raine-Fenning N, Deb S, Jayaprakasan K, Clewes J, Hopkisson J, Campbell B. Timing of oocyte maturation and egg collection during controlled ovarian stimulation: a randomized controlled trial evaluating manual and automated measurements of follicle diameter. Fertil Steril. 2010;94(1):184-8.

Rodrigues CL, Ziegelmann, PK. Metanálise: Um guia prático. Rev HCPA. 2010;30(4):436-47.

Roest J, Verhoeff A, van Heusden AM, Zeilmaker GH. Minimal monitoring of ovarian hyperstimulation: a useful simplification of the clinical phase of in vitro fertilization treatment. Fertil Steril. 1995;64(3):552-6.

Rongieres C. [Monitoring ovarian stimulation: are hormonal assessments necessary?]. J Gynecol Obstet Biol Reprod. 2006;35(5 Pt 2):2S39-2S41.

Strawn EY, Roesler M, Granlund A, Bohling S. In vitro fertilization can be successfully accomplished without routine estradiol monitoring: a randomized pilot study. Fertil Steril. 2007;88:S147.

Tang H, Hunter T, Hu Y, Zhai SD, Sheng X, Hart RJ. Cabergoline for preventing ovarian hyperstimulation syndrome. Cochrane Database Syst Rev. 2012;2:CD008605.

Vandekerckhove F, Vansteelandt S, Gerris J, De Sutter P. Follicle measurements using sonography-based automated volume count accurately predict the yield of mature oocytes in in vitro fertilization/intracytoplasmic sperm injection cycles. Gynecol Obstet Invest. 2013;76(2):107-12. 
Wiser A, Gonen O, Ghetler Y, Shavit T, Berkovitz A, Shulman A. Monitoring stimulated cycles during in vitro fertilization treatment with ultrasound only preliminary results. Gynecol Endocrinol. 2012;28(6):429-31.

Zegers-Hochschild F, Adamson GD, de Mouzon J, Ishihara O, Mansour R, Nygren $\mathrm{K}$, et al. International Committee for Monitoring Assisted Reproductive Technology (ICMART) and the World Health Organization (WHO) revised glossary of ART terminology, 2009. Fertil Steril. 2009;92(5):1520-4. 


\section{ANEXO DE PUBLICAÇÃO}


Ultrasound for monitoring controlled ovarian stimulation: a systematic review and meta-analysis of randomized controlled trials

Wellington P. MARTINS ${ }^{1,2}$, Clarissa V. R. VIEIRA ${ }^{1}$, Danielle M. TEIXEIRA ${ }^{1}$, Mariana A. P. BARBOSA ${ }^{1}$, Lilian A. DASSUNÇÃO ${ }^{1}$, Carolina O. NASTRI ${ }^{1,2}$.

${ }^{1}$ Department of Obstetrics and Gynecology, Medical School of Ribeirao Preto, University of Sao Paulo (DGO-FMRP-USP), Ribeirao Preto, Brazil.

${ }^{2}$ Ultrasonography and Retraining Medical School of Ribeirao Preto (EURP), Ribeirao Preto, Brazil

Correspondent author: Wellington P. Martins, Department of Obstetrics and Gynecology, Ribeirao Preto Medical School, University of São Paulo. Av. Bandeirantes, 3900 - $8^{\circ}$ andar - Monte Alegre. Ribeirao Preto; Sao Paulo; Brazil. 14048-900; Phone: +55(16)3602-2583; Fax: +55(16)3633-0946.

E-mail:wpmartins@gmail.com.

Keywords: Ultrasonography; Assisted reproduction techniques; Ovulation induction 


\section{Abstract}

Objectives: To evaluate the efficacy and safety of monitoring controlled ovarian stimulation by ultrasound. Methods: Authors searched for randomized controlled trials (RCT) in April 2013: studies that compared different methods for monitoring COS including ultrasound assessment of follicles (alone or combined with hormonal levels) in at least one group were considered eligible. Results: The search retrieved 1515 records; six studies were eligible. No study reported live birth. Five studies compared US only vs. US + Hormones: the confidence intervals $(\mathrm{CI})$ were compatible with appreciable benefit to appreciable harm of US Only for both OHSS (Odds Ratio 1.02, 95\% 0.47 to 2.25), and miscarriage (Risk Ratio 0.37, $95 \% \mathrm{Cl}$ 0.07 to 1.79 ). For clinical pregnancy, the $\mathrm{Cl}$ was compatible with small benefit to small harm (Risk Ratio $0.95,95 \% \mathrm{Cl} 0.78$ to 1.16 ). For the number of oocytes retrieved, the $\mathrm{Cl}$ was compatible with appreciable benefit to no effect (Mean Difference 0.77 oocytes, $95 \% \mathrm{Cl}-0.42$ to 1.96$)$. One study compared 3DUS vs. 2DUS: the $\mathrm{Cl}$ was compatible with appreciable benefit to appreciable harm of 3DUS regarding clinical pregnancy (Risk Ratio $1.00,95 \% \mathrm{Cl}=0.58$ to 1.73 ) and number of oocytes retrieved (Mean Difference -0.37 oocytes, $95 \% \mathrm{Cl}-3.63$ to 2.89 ). Conclusions: Monitoring COS with US only is unlikely to cause a relevant reduction in the number of oocytes retrieved and to substantially change the chance of clinical pregnancy when compared with US + Hormones; but we are uncertain of the effect on other important outcomes. We are also uncertain about the effect of using 3DUS for monitoring COS. 


\section{Introduction}

\section{Description of the condition}

Assisted reproductive techniques (ART) are widely used for the treatment of infertility/subfertility $(1,2)$. These procedures include in vitro handling of both human oocytes and sperm or of embryos with the objective of achieving pregnancy and live birth (3). In order to optimize ART results, the recruitment of multiple follicles is often necessary, and it's achieved by performing a controlled ovarian stimulation (COS) $(4,5)$. The estimated pregnancy rate per started cycle with standard COS is approximately $30 \%(6)$; when using minimal ovarian stimulation, the pregnancy rate per cycle is lower: about $10 \%$ (7). Such difference highlights the relevance of COS for the success of ART. However, when performing standard COS to obtain more follicles, there is an increased risk of ovarian hyperstimulation syndrome (OHSS) (8, 9).

\section{Description of the intervention and how intervention might work}

COS monitoring by ovarian follicles counting and measurement by ultrasonography and / or hormones dosage, particularly of serum estradiol concentration, are frequently used for monitoring COS. When monitoring COS, clinicians are able to decide for: 1. early cancelation of the cycles without proper ovarian response, avoiding an unnecessary waste of resources; 2 . the most appropriate time to trigger final follicular maturation, maximizing the number of retrieved mature oocytes; and 3. to assess the risk of OHSS, which is useful to define the use of some recent strategies, as using cabergoline (10) or the replacement of human chorionic gonadotropin (hCG) by gonadotropin-releasing hormone $(\mathrm{GnRH})$ agonist to trigger the final follicular maturation $(8,11)$. Combining ultrasonography and hormones assessment can potentially provide more information to the clinician, avoiding wrong decision-makings based in an error of one method. However, the need for intensive monitoring of COS is controversial: the combination of methods adds costs and discomfort for the woman who is undergoing ART and requires additional time. 
Three-dimensional ultrasonography (3DUS) is also being used for this purpose. The assessment of the follicles dimensions was described in mid-90s (12). More recently, the semi-automatic measurement of follicle volume or diameter by 3DUS - Sonography-based Automated Volume Count (SonoAVC) - is being investigated (13): this method was shown to have a good agreement with the conventional ultrasonography and require less time to be performed (14), being particularly advantageous when there are several follicles (15). Other potential advantages would be considering the volume-based diameter (helping reducing variability of measurements in non-spherical follicles) and avoiding double-counting the same follicles.

\section{Why it is important to do this review}

Given that the benefits of monitoring the COS are well established in the literature and its vigilance is practiced worldwide, one should seek the most appropriate method for such monitoring. So far, recommendation over this issue is ambiguous: in the last published systematic review and meta-analysis (16) the authors did not find evidence from randomized controlled trials supporting that combined monitoring for COS is more efficacious than cycle monitoring by ultrasound for live birth and clinical pregnancy; however, they suggested using combined monitoring for $\mathrm{COS}$ as a precautionary good practice since the evidence on the prevention of OHSS was not properly evaluated by the included RCTs. An update of the previous systematic review would also be interesting because new studies on this subject were published and the precision of the estimates could be improved.

The objective of this review is to evaluate the efficacy and safety of monitoring COS by ultrasound with or without hormonal levels for women undergoing ART and to compare different ultrasound methods. 


\section{Methods}

\section{Protocol and registration}

The

(http://www.crd.york.ac.uk/prospero/): CRD42012003297.

\section{Eligibility criteria}

Only truly randomized controlled trials (RCT) were considered eligible; quasi or pseudo randomized trials were not included $(17,18)$. Studies that permitted the inclusion of the same participant more than once (cross-over trials or "per cycle" studies) would be included only if: 1. data regarding the first cycle of each participant was available; or 2. if all consecutive cycles from each participant were performed in the same group: in all cases, the number of allocated women (not the number of cycles) would be considered as denominator. We included trials that compared different methods for monitoring COS including ultrasound assessment of follicles (alone or combined with hormonal levels) in at least one group.

\section{Information sources}

We searched trials using the following electronic databases: Cochrane Central Register of Controlled Trials (CENTRAL); Cumulative Index to Nursing and Allied Health Literature (CINAHL); Embase; Literatura Latino-Americana e do Caribe em Ciências da Saúde (LILACS); Medical Literature Analysis and Retrieval System Online (MEDLINE), and PsycINFO. Trials registers will be searched in Current Controlled Trials (www.controlled-trials.com), ClinicalTrials.gov (http://clinicaltrials.gov/ct2/search), and the World Health Organization International Trials Registry Platform search portal (http://apps.who.int/trialsearch/Default.aspx). In addition, authors hand-searched the reference list of the included articles and similar reviews in order to find additional records.

\section{Search}

The following search terms were used: (ultrasonography) OR (ultrasound) AND (ovarian stimulation) OR (ovarian hyperstimulation) OR (ovulation induction) 
OR (controlled ovarian) OR (IVF) OR (ICSI) OR (in vitro fertilization) OR (intracytoplasmic sperm injection) OR (assisted reproduction) OR (embryo transfer) OR (embryo deposition) OR (embryo replacement) OR (blastocyst) using filters for randomized controlled trials, and adjusting for each database as necessary. We did not impose any publication date or language limits for the searches.

\section{Trial selection}

Titles and abstracts were reviewed independently by two review authors (CVRV and LAD) checking for duplicates and using the pre-established criteria for inclusion. Disagreements were solved by consulting a third review author (WPM). These authors retrieved the full-text manuscripts of trials considered to be potentially eligible for inclusion. Two review authors (WPM and CON) independently evaluated eligibility of these trials. Disagreements would be solved by consensus. We corresponded with study investigators as required, to clarify study eligibility. There was no limitation on language, publication date or publication status.

\section{Data collection process}

Two authors (CON, WPM) independently extracted the data from included studies using a data extraction form planned and pilot-tested by the authors. Another author (DMT) checked the data. Disagreements would be solved by consensus. Authors corresponded with study investigators as required, to resolve data queries. In order to analyze data according to the intention-to-treat principle, the number of allocated women was used as the denominator as far as possible. The names of article authors and titles of the included studies were juxtaposed seeking for duplicate publication; duplicated publications were considered by the authors as being part of a unique study.

\section{Data items}

The following data were extracted from included studies: 1) methods: aim of trial, method of recruitment of participants, inclusion/exclusion criteria, and if informed consent was obtained as well as ethical approval; 2) participants characteristics: number, age, infertility cause; 3 ) intervention: the use of US and / or 
hormonal levels to monitor COS; 4) Primary outcomes: effectiveness - live birth per allocated women; adverse events: OHSS per allocated women; 5) Secondary outcomes: clinical pregnancy per allocated woman, miscarriage per clinical pregnancy, and number of retrieved oocytes (mature or total). Implantation rate was not included in the meta-analysis because the denominator for this outcome (number of embryos transferred) was not actually randomized; however, we planned to include this information in the characteristics of included studies.

\section{Risk of bias in individual studies}

Two reviewers (CON and WPM) independently assessed the risk of selection bias (random sequence generation and allocation concealment); performance bias (blinding of participants and personnel); detection bias (blinding of outcome assessors); attrition bias (incomplete outcome data); reporting bias (selective outcome reporting); and other potential sources of bias (number of embryos transferred). To judge the risk of bias, we used the Cochrane Collaboration's criteria for judging risk of bias (19); the studies were classified as being at 'low', high' or 'unclear' risk of bias.

\section{Summary measures}

All results were combined for meta-analysis with Review Manager 5.1 (Copenhagen: The Nordic Cochrane Centre, The Cochrane Collaboration, 2011). The numbers of events in the control and intervention groups of each study were used to calculate Mantel-Haenszel risk ratio (RR). We prefer to use RR because odds ratio (OR) is harder to understand and to apply in practice; additionally there is some concern that OR might be misinterpreted as if it equated to the $R R$, which will tend to overestimate the intervention effect, especially when events are common (19). However, when a zero cell count or prevalence $<1 \%$ was observed, the Peto fixed-effect OR was be used, because this method is found to be the least biased and most powerful, providing the best confidence interval coverage in these situations (19); additionally, the resulting OR value in such situations is very similar to $\mathrm{RR}$, avoiding misinterpretations. The precision of the estimates were evaluated 
by the $95 \%$ confidence interval. We considered the clinical relevance of any statistically significant findings.

\section{Synthesis of results}

The following comparisons were planned: 1) Ultrasound only vs. Ultrasound + Hormones; 2) Ultrasound + Hormones vs. Hormones only; 3) Ultrasound only vs. Hormones only; 4) 3DUS (including semiautomatic method) with or without Hormones vs. 2DUS with or without Hormones. An increase in the risk of a particular outcome associated with the use of ultrasound, which may be beneficial (e.g. live birth) or detrimental (e.g. OHSS), was displayed graphically in the metaanalyses to the right of the center-line, while a decrease in the risk of an outcome to the left of the center-line. We assessed heterogeneity by using $\mathrm{I}^{2}$. Substantial heterogeneity $\left(I^{2}>50 \%\right)$ among the studies would be addressed firstly by checking again that the data is correct and, secondly, by using a random-effect model.

\section{Risk of bias across studies}

In view of the difficulty of detecting and correcting for publication bias and other reporting biases, the authors aimed to minimize their potential impact by ensuring a comprehensive search for eligible studies and by being alert for duplication of data. If there were ten or more studies in an analysis, we had planned to use a funnel plot to explore the possibility of small study effects (a tendency for estimates of the intervention effect to be more beneficial in smaller studies).

\section{Additional analysis}

No subgroup analysis was planned. Sensitivity analysis was planned to be performed in order to verify whether the conclusions would differ if eligibility was restricted to studies judged to no be at a high risk of bias.

\section{Overall quality of the body of evidence: Summary of Findings Table}

A summary of findings table was generated using GRADEPRO software. The quality of the evidence for the main review outcomes was evaluated using the following GRADE criteria: study limitations (i.e. risk of bias), consistency of effect, 
imprecision, indirectness and publication bias. Judgments about evidence quality (high, moderate, low or very low) are justified, documented, and incorporated into reporting of results for each outcome (20).

\section{Results}

\section{Study selection}

The electronic search was run in Apr-15-2013 and a total of 1,515 records were retrieved: $\mathrm{CENTRAL}=302 ; \mathrm{CINAHL}=120 ;$ Embase $=343 ;$ LILACS $=4$; MEDLINE = 612; PsycINFO = 8; ClinicalTrials = 65; Controlled-trials $=27 ;$ WHO International Trials Registry Platform $=34$. One additional record was retrieved through manual search of reference lists of potentially eligible studies and related reviews. After removing duplicates, 1,126 records were screened on the basis of title and abstracts; 11 records were considered as potentially eligible and further evaluated for eligibility. From these records, 4 studies were excluded with reasons: two studies were not randomized $(21,22)$, one study was pseudo-randomized (15), and one record was related to an ongoing trial that is still recruiting participants (23); 6 studies from 7 records were included in the quantitative meta-analysis (Figure 1).

\section{Study characteristics}

Six studies were included in the quantitative analysis and their characteristics are reported in Table 1. All studies were parallel-design RCTs. Five studies were single centered: 2 in Israel $(24,25) 1$ in Spain (26), 1 in the United Kingdom (27), and 1 in France (28). The other study was conducted in four centers in the United Kingdom (29). We contacted the corresponding authors to obtain further information of two studies $(25,29)$.

\section{Participants}

A total of 797 women from six studies were included; 36 were conducted only with 3DUS, 404 only with 2DUS and 357 with US and hormonal levels. In one study, women were allowed to perform additional cycles in the same assigned group (26): 42 cycles from 31 women (average $=1.35$ cycles per woman) in one 
group, and 48 cycles from 35 women (average $=1.37$ cycles per woman) in the other.

\section{Interventions}

Five studies compared US only with US and hormonal levels for COS (24-26, $28,29)$. One study (27) compared 3DUS with 2DUS for COS: hormones were not assessed in any group. In three studies, women monitored with US only had a blood estradiol dosage just before inducing the final follicular maturation with hCG (24-26).

\section{Outcomes}

No study reported live birth; 4/6 reported OHSS; 5/6 reported clinical pregnancy; $1 / 6$ reported miscarriage; $5 / 6$ reported total oocytes retrieved. No study reported implantation rate.

\section{Risk of bias within studies}

Five studies applied adequate methods of randomization, and in one the method for randomization was not described (24). In four studies, the method for allocation concealment was not clear; these were deemed at unclear risk of selection bias $(24,26-28)$. We did not consider that blinding was likely to influence the risk of performance bias for the present review. Two studies were deemed at high risk of attrition bias because of some participants were excluded from analysis $(25,26)$ : in one study, four women were excluded from analysis because they did not follow the study protocol (26); in other study two women were excluded after allocation (25): one became pregnant before COS and the other left the clinic before starting treatment. One study was considered at unclear risk of reporting bias (24): the authors only reported the proportion of clinical pregnancies per oocyte retrieval and per embryo transfer, but they did not report the number of women submitted to oocyte retrieval and/or embryo transfer in each group; and therefore, we could not determine the total number of clinical pregnancies in each group. One study was deemed at high risk of other bias because the initial intervention proposed to the US + Hormones group was modified during the trial: 40/148 women 
did not achieve the criteria for hCG administration using the initial proposed criteria, but they received hCG anyway, following the criteria proposed for the US only group (29). No study reported significant differences in the baseline age, BMI, or FSH between the groups. The risk of bias summary is presented in Figure 2.

\section{Synthesis of results}

Comparison: Ultrasound only vs. Ultrasound + Hormones

1. Live birth per allocated woman

No study reported this outcome.

\section{OHSS}

The $\mathrm{Cl}$ was very wide and compatible with appreciable benefit, no effect or appreciable harm for US only compared with US + Hormones: OR 1.02, 95\% CI 0.47 to $2.25, \mathrm{P}=0.95,5 \mathrm{RCTs}, 725$ women, $\mathrm{I}^{2}=0$, low-quality evidence (Figure 3 ). When performing the sensitivity analysis, restricting eligibility criteria only to studies judged to not be at a high risk of bias, the $\mathrm{Cl}$ was very wide and compatible with appreciable benefit, no effect or appreciable harm for US only compared with US + Hormones: OR $0.78,95 \% \mathrm{Cl} 0.27$ to $2.28, \mathrm{P}=0.65,2 \mathrm{RCTs}, 299$ women.

\section{Clinical pregnancy per allocated woman}

The $\mathrm{Cl}$ was wide and compatible with small benefit, no effect or small harm for US only compared with US + Hormones: RR $0.95,95 \% \mathrm{Cl} 0.78$ to $1.16, \mathrm{P}=0.61$, 4 RCTs, 611 women, $I^{2}=10 \%$, low-quality evidence (Figure 4). When performing the sensitivity analysis, restricting eligibility criteria only to studies judged to not be at a high risk of bias, the $\mathrm{Cl}$ was very wide and compatible with appreciable benefit, no effect or appreciable harm for US only compared with US + Hormones: RR 0.96, $95 \% \mathrm{Cl} 0.62$ to $1.48, \mathrm{P}=0.84,1 \mathrm{RCT}, 185$ women.

\section{Miscarriage per clinical pregnancy}

Only one study reported miscarriage, and the $\mathrm{Cl}$ was very wide: the findings were compatible with appreciable benefit, no effect or appreciable harm for US only 
compared with US + Hormones: $\mathrm{RR} 0.37,95 \% \mathrm{Cl} 0.07$ to $1.79, \mathrm{P}=0.21,45$ pregnant women, very-low quality evidence. Sensitivity analysis was not performed because the only study that reported miscarriage was considered to be at a high risk of bias.

\section{Total number of oocytes retrieved}

The $\mathrm{Cl}$ was wide and compatible with appreciable benefit or no effect for US only compared with US + Hormones: MD 0.77 oocytes, $95 \% \mathrm{Cl}-0.42$ to 1.96 , $\mathrm{P}=0.20$, 3 RCTs, 474 women, $\mathrm{I}^{2}=0$, moderate-quality evidence (Figure 5). When performing the sensitivity analysis, the $\mathrm{Cl}$ was wide and compatible with appreciable benefit, no effect, or small harm for US only compared with US + Hormones: MD 1.70 oocytes, $95 \% \mathrm{Cl}-1.22$ to $4.62, \mathrm{P}=0.25,1 \mathrm{RCT}, 114$ women.

\section{Comparison: 3DUS vs. 2DUS}

There was only one study included in this comparison; this study did not report live birth, OHSS, or miscarriage. For clinical pregnancy the $\mathrm{Cl}$ was very wide and compatible with appreciable benefit, no effect or appreciable harm for 3DUS compared with 2DUS: $\mathrm{RR} 1.00,95 \% \mathrm{Cl} 0.58$ to $1.73, \mathrm{P}=1.00,72$ women. For the total number of oocytes retrieved the $\mathrm{Cl}$ was very wide and compatible with appreciable benefit, no effect or appreciable harm for 3DUS compared with 2DUS: MD -0.37 oocytes, $95 \% \mathrm{Cl}-3.63$ to $2.89, \mathrm{P}=0.82,72$ women. This study was not judged to be at a high risk of bias.

\section{Risk of bias across studies}

We did not identify any evidence of publication bias or selective reporting within studies. Since less than 10 studies were included, we did not perform funnel plot analysis.

\section{Additional analysis}

Sensitivity analysis, restricting the inclusion criteria to studies judged not to be at a high risk of bias, was the only additional analysis performed. This analysis was reported along with the main results. 


\section{Discussion}

\section{Summary of evidence}

Five studies were included for the comparison US only vs. US + hormones (Table 2). No study evaluated live birth. We observed moderate quality evidence that monitoring COS with US Only is unlikely to cause substantial reduction of the number of oocytes retrieved when compared with US + Hormones. There is low quality evidence that monitoring COS with US Only probably do not substantially change the chance of achieving a clinical pregnancy. We are still uncertain of the effect on OHSS and miscarriage. Only one study was included for the comparison 3DUS vs. 2DUS. This study provided evidence for the number of oocytes retrieved and clinical pregnancy; however, the estimates were not precise enough to rule out appreciable harm or benefit.

\section{Quality of the evidence}

In the comparison "US only vs. US + Hormones" (Table 2), the quality of the evidence for OHSS was considered to be low: it was downgraded one level because of imprecision (wide $95 \% \mathrm{Cl}$ ) and another level because of the high risk of bias observed in the included studies. The quality of the evidence for clinical pregnancy was also considered to be low: it was downgraded one level because of imprecision and another level because of the high risk of bias. The quality of the evidence for miscarriage was considered to be very low: it was downgraded two levels because of serious imprecision (very wide $95 \% \mathrm{Cl}$ ) and another level because of the high risk of bias. The quality of the evidence for the number of oocytes retrieved was considered to be moderate: it was downgraded one level because of the high risk of bias observed in the included studies. In the comparison "3DUS vs. 2DUS" (Table 2), the quality of the evidence for the two reported outcomes (clinical pregnancy and number of oocytes retrieved) was considered to be low: it was downgraded two levels because of serious imprecision (very wide $95 \% \mathrm{Cl})$. 


\section{Overall completeness and applicability of evidence}

There is another systematic review addressing this issue published in 2008 (16); at that time only two studies were included, and both are also included in the present review $(24,29)$. They concluded the evidence was too scarce at that point to draw any conclusion. We included more four studies: one from 2006 (64), and other three studies published after the former review (25-27).

Besides the 6 studies included in the quantitative review, three other studies addressed the review question but were excluded because they were not randomized $(15,21,22)$. Two of these studies $(21,22)$ evaluated the comparison US only vs. US + Hormones. One of them (22) reported live birth (RR 1.05, 95\% Cl 0.47 to $2.32, \mathrm{P}=0.91,206$ women); OHSS (OR $0.87,95 \% \mathrm{Cl} 0.05$ to $14.12, \mathrm{P}=0.92$, 206 women); and clinical pregnancy per woman allocated (RR $1.04,95 \% \mathrm{Cl} 0.57$ to $1.90, P=0.91,206$ women). The other (21) reported clinical pregnancy per allocated woman ( $\mathrm{RR} 0.83,95 \% \mathrm{Cl} 0.25$ to $2.80, \mathrm{P}=0.77,120$ women); and total number of oocytes retrieved (MD $-0.30,95 \% \mathrm{Cl}-1.63$ to $1.03, \mathrm{P}=0.66,120$ women). The third study (15) evaluated the use of the software SonoAVC under the comparison "US $3 \mathrm{D}$ vs. US $2 \mathrm{D}$ " and reported: clinical pregnancy per allocated woman (RR 0.90, $95 \% \mathrm{Cl} 0.47$ to $1.73, \mathrm{P}=0.75,40$ women); and total number of oocytes retrieved (MD 3.20 oocytes, $95 \% \mathrm{Cl}-0.89$ to $7.29, \mathrm{P}=0.12,40$ women). None of these three studies observed any significant difference between groups, which is in agreement with the result of the meta-analysis of the studies included in the quantitative review.

Monitoring COS with US + hormones results in increased costs and will also cause discomfort and stress related to the process of blood collection. Although we are uncertain of the effect for other important outcomes, monitoring COS with US only is not likely to reduce the number of oocytes retrieved or to cause substantial changes in the chance of achieving a clinical pregnancy. Therefore, we believe that US only should be considered as a reasonable alternative for monitoring COS.

\section{Limitations}

Although the included studies were consistent in the estimate of effect, the quality of the evidence compiled in this review was impaired by the studies' quality. 
Three out of six studies were deemed at high risk of bias. Despite the core reasons for eliminating some hormonal dosages and inclusion of semiautomatic techniques are the reduction of costs and/or stress, and the improvement of logistics; no trial studied the cost-effectiveness and/or the quality of life measure related to these interventions. Additionally, the small number of RCTs makes the assessment of publication and reporting bias sub-optimal, because of the impossibility of performing a funnel plot analysis.

\section{Conclusions}

Monitoring COS with US only is feasible and it is unlikely to cause a relevant reduction the number of oocytes retrieved; as well as substantial changes in clinical pregnancy when compared with US + Hormones. However, we are still uncertain of the effect for other important outcomes. We are also uncertain about the effect of using 3DUS for monitoring COS. More studies evaluating the optimal monitoring for COS are still needed. 


\section{Footnotes}

Authorship: Conception and design (CON, WPM); drafting the protocol (CVRD, DMT, LAD, MAPB, WPM); search strategy (WPM); study selection (CON, CVRD, LAD, WPM); data collection (CON, DMT, WPM); data analysis and interpretation (CON, DMT, WPM); writing the article (CON, DMT, WPM); revising it for important intellectual content (CVRD, LAD, MAPB). All authors had full access to all the data in the study and approved the final manuscript.

Funding: During this review, the authors received financial support from: CAPES, Brazil (CON); CNPq, Brazil (WPM); FAPESP, Brazil (DMT); HC-FMRP-USP, Brazil (WPM); EURP, Brazil (CON, WPM). These institutions had no influence on the study design, interpretation of data, or the decision to publish the results.

Competing interests: All authors declare no relationships or activities that could appear to have influenced the submitted work.

Ethical approval: This review was approved by our department's research committee. 


\section{References}

1. Gnoth C, Godehardt E, Frank-Herrmann P, Friol K, Tigges J, Freundl G. Definition and prevalence of subfertility and infertility. Hum Reprod 2005; 20 : 1144-1147.

2. de Mouzon J, Lancaster P, Nygren KG, Sullivan E, Zegers-Hochschild F, Mansour R, Ishihara O, Adamson D. World collaborative report on Assisted Reproductive Technology, 2002. Hum Reprod 2009; 24: 2310-2320.

3. Zegers-Hochschild F, Adamson GD, de Mouzon J, Ishihara O, Mansour R, Nygren K, Sullivan E, Vanderpoel S. International Committee for Monitoring Assisted Reproductive Technology (ICMART) and the World Health Organization (WHO) revised glossary of ART terminology, 2009. Fertil Steril 2009; 92: 1520-1524.

4. Figueiredo JB, Nastri CO, Vieira AD, Martins WP. Clomiphene combined with gonadotropins and $\mathrm{GnRH}$ antagonist versus conventional controlled ovarian hyperstimulation without clomiphene in women undergoing assisted reproductive techniques: systematic review and meta-analysis. Arch Gynecol Obstet 2013; 287: 779-790.

5. Martins WP, Vieira AD, Figueiredo JB, Nastri CO. FSH replaced by low-dose hCG in the late follicular phase versus continued FSH for assisted reproductive techniques. Cochrane Database Syst Rev 2013; 3: CD010042.

6. Gnoth C, Maxrath B, Skonieczny T, Friol K, Godehardt E, Tigges J. Final ART success rates: a 10 years survey. Hum Reprod 201; 26: 2239-2246.

7. Pelinck MJ, Vogel NE, Hoek A, Simons AH, Arts EG, Mochtar $M H$, Beemsterboer S, Hondelink MN, Heineman MJ. Cumulative pregnancy rates after three cycles of minimal stimulation IVF and results according to subfertility diagnosis: a multicentre cohort study. Hum Reprod 2006; 21: 2375-2383.

8. Nastri CO, Ferriani RA, Rocha IA, Martins WP. Ovarian hyperstimulation syndrome: pathophysiology and prevention. J Assist Reprod Genet 2010; 27: 121-128.

9. Cantwell R, Clutton-Brock T, Cooper G, Dawson A, Drife J, Garrod D, Harper A, Hulbert D, Lucas S, McClure J, Millward-Sadler H, Neilson J, Nelson-Piercy C, Norman J, O'Herlihy C, Oates M, Shakespeare J, de Swiet M, Williamson C, 
Beale V, Knight M, Lennox C, Miller A, Parmar D, Rogers J, Springett A. Saving Mothers' Lives: Reviewing maternal deaths to make motherhood safer: 20062008. The Eighth Report of the Confidential Enquiries into Maternal Deaths in the United Kingdom. BJOG 2011; 118 Suppl 1: 1-203.

10. Tang $\mathrm{H}$, Hunter $\mathrm{T}, \mathrm{Hu} \mathrm{Y}$, Zhai SD, Sheng X, Hart RJ. Cabergoline for preventing ovarian hyperstimulation syndrome. Cochrane Database Syst Rev 2012; 2: CD008605.

11. Humaidan P, Papanikolaou EG, Kyrou D, Alsbjerg B, Polyzos NP, Devroey P, Fatemi HM. The luteal phase after $\mathrm{GnRH}$-agonist triggering of ovulation: present and future perspectives. Reprod Biomed Online 2012; 24: 134-141.

12. Kyei-Mensah A, Zaidi J, Pittrof R, Shaker A, Campbell S, Tan SL. Transvaginal three-dimensional ultrasound: accuracy of follicular volume measurements. Fertil Steril 1996; 65: 371-376.

13. Raine-Fenning N, Jayaprakasan K, Clewes J, Joergner I, Bonaki SD, Chamberlain S, Devlin L, Priddle H, Johnson I. SonoAVC: a novel method of automatic volume calculation. Ultrasound Obstet Gynecol 2008; 31: 691-696.

14. Ata B, Seyhan A, Reinblatt SL, Shalom-Paz E, Krishnamurthy S, Tan SL. Comparison of automated and manual follicle monitoring in an unrestricted population of 100 women undergoing controlled ovarian stimulation for IVF. Hum Reprod 2011; 26: 127-133.

15. Murtinger M, Aburumieh A, Rubner $\mathrm{P}$, Eichel V, Zech $\mathrm{MH}$, Zech $\mathrm{NH}$. Improved monitoring of ovarian stimulation using $3 \mathrm{D}$ transvaginal ultrasound plus automated volume count. Reprod Biomed Online 2009; 19: 695-699.

16. Kwan I, Bhattacharya S, McNeil A, van Rumste MM. Monitoring of stimulated cycles in assisted reproduction (IVF and ICSI). Cochrane Database Syst Rev 2008; CD005289.

17. Martins WP, Rocha IA, Ferriani RA, Nastri CO. Assisted hatching of human embryos: a systematic review and meta-analysis of randomized controlled trials. Hum Reprod Update 2011; 17: 438-453.

18. Nastri CO, Gibreel A, Raine-Fenning N, Maheshwari A, Ferriani RA, Bhattacharya S, Martins WP. Endometrial injury in women undergoing assisted reproductive techniques. Cochrane Database Syst Rev 2012; 7: CD009517. 
19. Higgins J, Green S. (editors) Cochrane Handbook for Systematic Reviews of Interventions Version 5.1.0 [updated March 2011]. The Cochrane Collaboration.

20. Guyatt G, Oxman AD, Akl EA, Kunz R, Vist G, Brozek J, Norris S, Falck-Ytter Y, Glasziou P, DeBeer H, Jaeschke R, Rind D, Meerpohl J, Dahm P, Schunemann HJ. GRADE guidelines: 1. Introduction-GRADE evidence profiles and summary of findings tables. J Clin Epidemiol 2011; 64: 383-394.

21. Kably Ambe A, Carranza Lira S, Serviere Zaragoza C, Espinoza de los Monteros A, Coria Soto I, Alvarado Duran A. The evaluation of the usefulness of ultrasound independent of estradiol as a prognostic criterion for ovular capture and maturity. A comparative double-blind study. Ginecol Obstet Mex 1994; 62: 288-291.

22. Murad NM. Ultrasound or ultrasound and hormonal determinations for in vitro fertilization monitoring. Int J Gynaecol Obstet 1998; 63: 271-276.

23. Ben-Haroush A. Automated 3D (SonoAVC) Versus 2D Ovarian Follicles Assessment in IVF Cycles: a Randomized Controlled Trial. NCT01723267. 2012.

24. Golan A, Herman A, Soffer Y, Bukovsky I, Ron-El R. Ultrasonic control without hormone determination for ovulation induction in in-vitro fertilization/embryo transfer with gonadotrophin-releasing hormone analogue and human menopausal gonadotrophin. Hum Reprod 1994; 9: 1631-1633.

25. Wiser A, Gonen O, Ghetler Y, Shavit T, Berkovitz A, Shulman A. Monitoring stimulated cycles during in vitro fertilization treatment with ultrasound only-preliminary results. Gynecol Endocrinol 2012; 28: 429-431.

26. Aguirre SG, Hevia IG, García-Carpintero G, Rosal MJ. Measuring plasma estradiol levels in in vitro fertilization. Is it useful? Rev Iberoam Fertil Reprod Hum 2010; 27: 43-49.

27. Raine-Fenning N, Deb S, Jayaprakasan K, Clewes J, Hopkisson J, Campbell B. Timing of oocyte maturation and egg collection during controlled ovarian stimulation: a randomized controlled trial evaluating manual and automated measurements of follicle diameter. Fertil Steril 2010; 94: 184-188.

28. Rongieres C. Monitoring ovarian stimulation: are hormonal assessments necessary? J Gynecol Obstet Biol Reprod 2006; 35: 2S39-32S41. 
29. Lass A. Monitoring of in vitro fertilization-embryo transfer cycles by ultrasound versus by ultrasound and hormonal levels: a prospective, multicenter, randomized study. Fertil Steril 2003; 80: 80-85. 
Figures Legend

Figure 1: Flowchart of study selection.

Figure 2: Risk of bias summary: review authors' judgements about each risk of bias item for each included study.

Figure 3: Ovarian hyperstimulation syndrome (OHSS) - forest plot; comparison US only versus US + Hormones.

Figure 4: Clinical pregnancy - forest plot; comparison US only versus US + Hormones.

Figure 5: Oocytes retrieved - forest plot; comparison US only versus US + Hormones. 


\section{Figure 1}

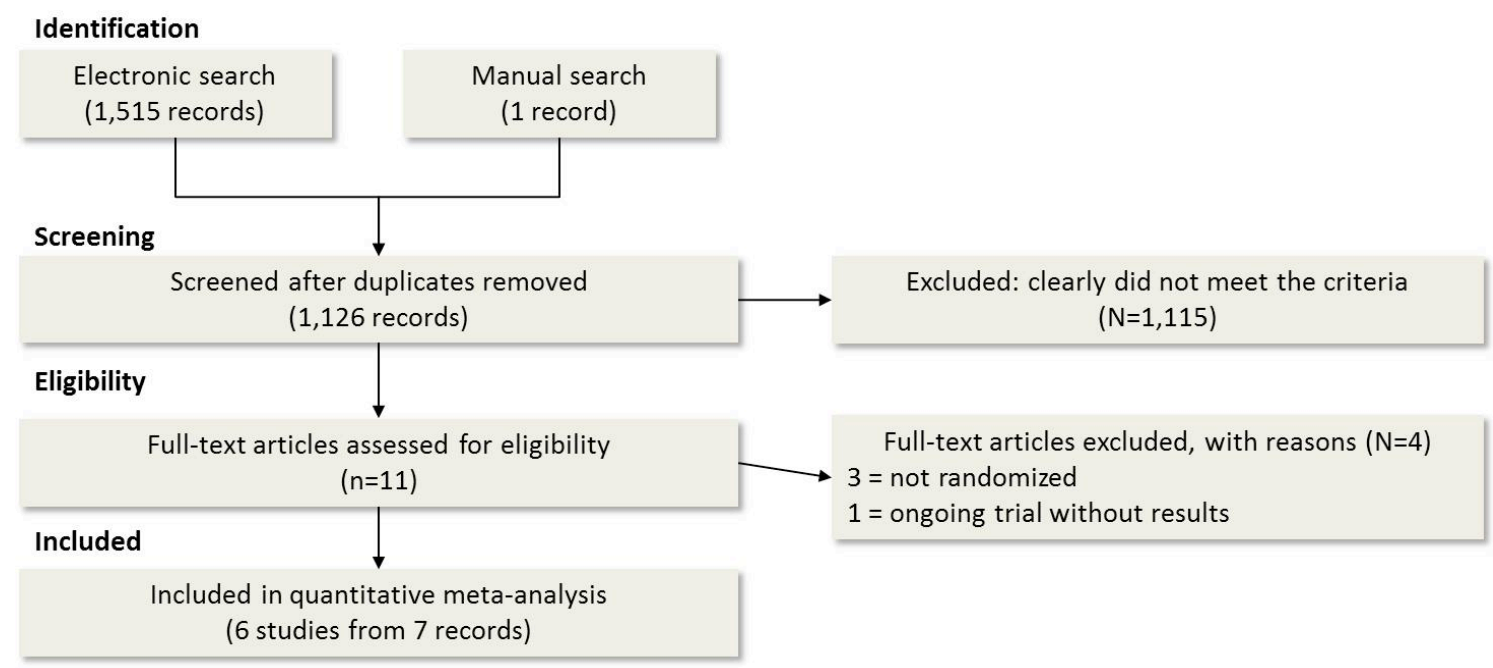


Figure 2

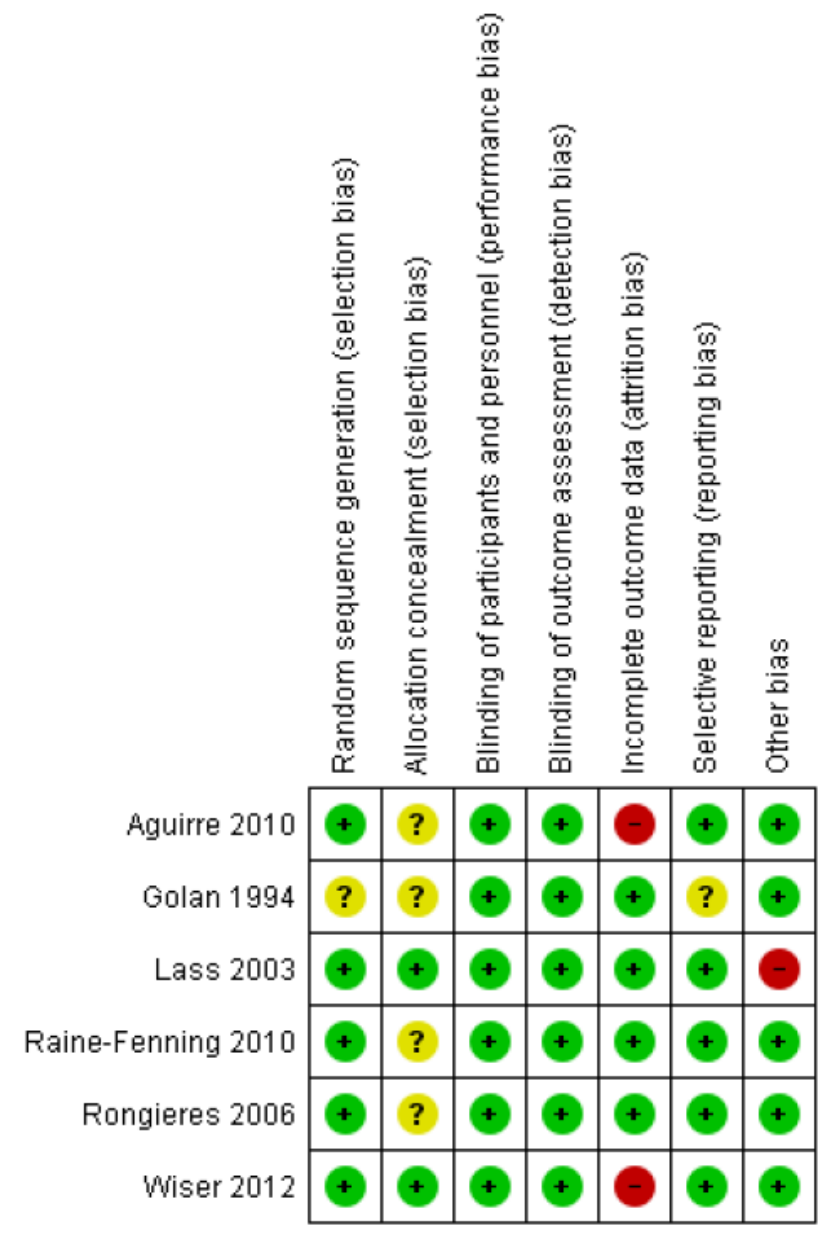

Figure 3

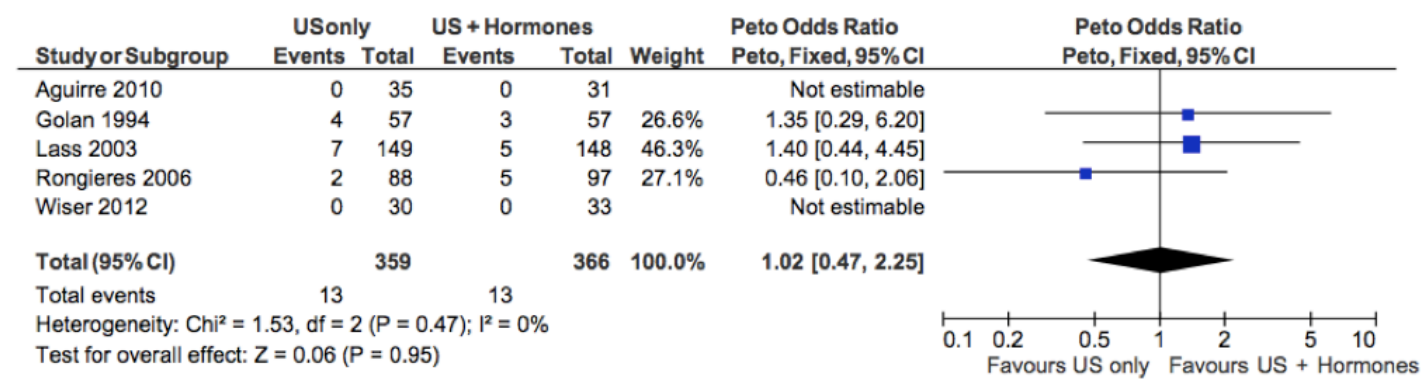


Figure 4

\begin{tabular}{|c|c|c|c|c|c|c|c|c|c|}
\hline Study or Subgroup & \multicolumn{2}{|c|}{ USonly } & \multicolumn{2}{|c|}{ US + Hormones } & Weight & $\begin{array}{c}\text { Risk Ratio } \\
\text { M-H,Fixed, } 95 \% \mathrm{Cl}\end{array}$ & \multicolumn{2}{|r|}{$\begin{array}{c}\text { Risk Ratio } \\
\text { M-H,Fixed, } 95 \% \mathrm{Cl}\end{array}$} & \\
\hline Aguirre 2010 & 26 & 35 & 19 & 31 & $17.4 \%$ & $1.21[0.86,1.70]$ & & & \\
\hline Lass 2003 & 46 & 149 & 49 & 148 & $42.4 \%$ & $0.93[0.67,1.30]$ & & & \\
\hline Rongieres 2006 & 26 & 88 & 30 & 97 & $24.6 \%$ & $0.96[0.62,1.48]$ & & & \\
\hline Wiser 2012 & 12 & 30 & 19 & 33 & $15.6 \%$ & $0.69[0.41,1.18]$ & & & \\
\hline Total $(95 \% \mathrm{Cl})$ & & 302 & & 309 & $100.0 \%$ & $0.95[0.78,1.16]$ & & & \\
\hline Total events & 110 & & 117 & & & & & & \\
\hline $\begin{array}{l}\text { Heterogeneity: } \mathrm{Chi}^{2}= \\
\text { Test for overall effect }\end{array}$ & $\begin{array}{l}3.33, \mathrm{df}= \\
Z=0.50\end{array}$ & $\begin{array}{l}3(P= \\
P=0.6\end{array}$ & $\begin{array}{l}0.34) ;\left.\right|^{2}=1 \\
\text { 1) }\end{array}$ & & & & $\begin{array}{ll}0.1 & 0.2 \\
\text { ours US }\end{array}$ & $\begin{array}{ccc}0.5 & 1 & 2 \\
\text { Hormones } & \text { Favours }\end{array}$ & $\begin{array}{cc}5 & 10 \\
\text { only } & \end{array}$ \\
\hline
\end{tabular}

\section{Figure 5}

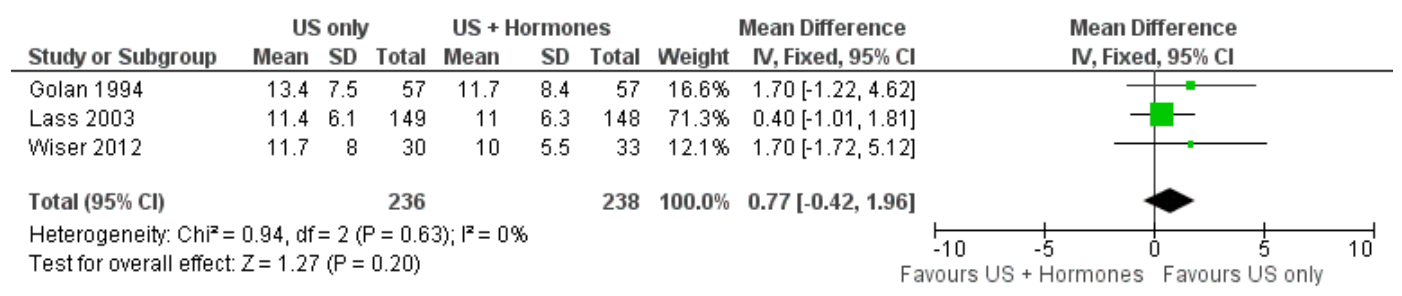

